\title{
Medicinal and aromatic species of Asteraceae commercialized in the conurbation Buenos Aires-La Plata (Argentina)
}

\author{
Julio Alberto Hurrell ${ }^{1,2,}$ and Jeremías Pedro Puentes ${ }^{1,3}$ \\ ${ }^{1}$ Laboratorio de Etnobotánica y Botánica Aplicada (LEBA), Facultad de Ciencias Naturales y Museo, \\ Universidad Nacional de La Plata. Calle 64 no. 3, 1900-La Plata, Argentina. \\ ${ }^{2}$ Consejo Nacional de Investigaciones Científicas y Técnicas (CONICET), Argentina. \\ ${ }^{3}$ Comisión de Investigaciones Científicas (CIC), Provincia de Buenos Aires, Argentina. \\ * Corresponding author \\ \E-mail adresses: JAH (juliohurrell@gmail.com), JPP: (jeremiasppuentes@gmail.com)
}

Ethnobiology and Conservation 2013, 2:7 (01 September 2013)

ISSN 2238-4782

doi: 10.15451/ec2013-8-2.7-1-40

ethnobioconservation.com

\section{Abstract}

The present contribution includes 50 species of Asteraceae whose products are marketed with therapeutic and aromatic purposes in the conurbation Buenos AiresLa Plata, the largest metropolitan area of Argentina. For each species, the scientific and vernacular names, types of products and samples are given. The plant products comprise fresh plants or parts thereof, herbal products, tinctures, and dietary supplements, among others. The uses assigned and the effects scientifically studied are indicated. Also, the ethnobotanical value of the diverse plant products and their commercial circulation are discussed. Circulating products and their uses were studied, both in the restricted context of immigrant segments (Bolivian and Chinese), and in the broader context of general commercial circuit (health food stores, named locally 'dietéticas'). Botanical knowledge of immigrants segments is considered linked to their traditions, and botanical knowledge of general commercial circuit is regarded nontraditional. Research conducted is framed within the urban ethnobotany context. Specially, it addresses some relevant theoretical and methodological topics within discipline: composition of botanical knowledge in urban pluricultural scenarios (linked to traditions, nontraditional), the embodiment of this knowledge in actions (such as selection of products to consume), and the dynamic of changes in urban botanical knowledge (dispersion of products and uses in the commercial circuit and media).

Keywords: Urban ethnobotany - Asteraceae - Conurbation Buenos Aires-La Plata, Argentina 
Hurrell and Puentes 2013. Medicinal and aromatic species of Asteraceae commercialized in the conurbation Buenos Aires-La Plata (Argentina). Ethnobio Conserv 2:7

\section{Introduction}

This contribution presents partial results obtained from a research line about urban ethnobotany, carried out at the Laboratorio de Etnobotánica y Botánica Aplicada (LEBA), Facultad de Ciencias Naturales y Museo, Universidad Nacional de La Plata, Argentina. This research line addresses the study of composition and dynamics of the urban botanical knowledge, a central issue of urban ethnobotany because that knowledge guides the selection of plants, parts thereof and products derived from them in urban pluricultural contexts. The study area comprises the conurbation Buenos Aires-La Plata, the largest metropolitan area of Argentina both in extension and population. It includes two contiguous urban agglomerations: one emerged around Buenos Aires, the capital city of the country, the other around La Plata, the capital city of Buenos Aires province.

The results presented here correspond to 50 species of Asteraceae marketed as medicinal and/or aromatic in the conurbation. Asteraceae is the family of flowering plants with greater diversity worldwide: 1,600 genera and 23,600 species, distributed in all continents, except Antarctica (Stevens 2013), and its economical and medicinal importance has been widely described (Heywood et al. 1977). Asteraceae is the largest plant family in Argentina, with 227 genera and around 1,400 native and adventitious species (Katinas et al. 2007), also it has the greatest number of native taxa used in popular medicine in this country (more than 270 species), followed by Leguminosae and Solanaceae (Barboza et al. 2009).

\section{Framework}

Ethnobotany is the study of the complex relations between people and plants, based on the theoretical context of ecology (Albuquerque and Hurrell 2010; Hurrell and Albuquerque 2012). An aspect related to those relationships has acquired a special development: studies about botanical knowledge (BK), that it is defined as an ensemble of knowledge and beliefs that people have about the vegetal elements of their surroundings: plants, parts thereof, or products deriving from them (Hurrell et al. 2011a). The BK orients diverse behaviors, like agricultural practices in rural contexts, or selecting vegetal elements consumed in urban scenarios. When we say: 'knowledge orients the actions', we mean that knowledge is embodied in diverse behaviors that become adaptive. In the ethnobotanical work is expected to extrapolate the underlying knowledge from the actions. For example: Why a plant is used in one way and not another? Or: Why a particular use may change over time? The called embodiment of knowledge (Martínez 2008; Varela 1990) is a complex concept. In our theoretical framework, we assume that knowledge generates actions, but we also must consider that, in turn, the actions feeds back on knowledge, which generates new patterns of action, and so on. So, the human-plant system evolves. This is a basic premise for ongoing investigations.

Most of the researches on BK are oriented to traditional botanical knowledge (TBK), the knowledge and beliefs of culturally homogeneous contexts where there is a direct link between production and consumption: 'those who consume, produce'. The BK of urban agglomerations is considered nontraditional in opposition to the TBK: it corresponds to pluricultural contexts where there is an indirect link between production and consumption: 'those who consume do not produce'. This type of BK is likewise adaptive, because it guides the election of the products to consume, while others are discarded (Pochettino et al. 2012a). But urban botanical knowledge (UBK) is more than nontraditional knowledge. UBK is a whole composed of different knowledges about plants and its derived products that coexist and interact within the 
same pluricultural frame. The entire population of the metropolitan areas is highly heterogeneous, and brings together local inhabitants as well as diverse groups of immigrants from different origins and residence time in the area. Local inhabitants have a mostly nontraditional BK (including scientific knowledge), although many retain certain knowledge linked to their own family traditions. The different segments of immigrants preserve knowledge associated with their traditions of origin. The BK of these segments is linked to traditions, but it does not strictly a TBK, because it is readapted to a new cultural context. While some ethnobotanical researches deal with plants product used by the average consumer segment in urban scenarios (Arenas et al. 2011; Ladio and Molares 2010; Pochettino et al. 2008), most of the papers are devoted to groups of immigrants that preserve a BK linked to their native traditions (Ceuterick et al. 2008; Pieroni et al. 2005; Pochettino et al. 2012a; Sandhu and Heinrich 2005; Volpato et al. 2009).

Consequently, urban ethnobotany must to give answers to two key questions: 1. How is the UBK composition? That is: linked to traditions, nontraditional. 2. How is the UBK dynamics? That is how the transmission of knowledge about vegetal elements and their uses take place in the urban pluricultural context. Several plants, their parts and products are visible for everyone and belong to the general commercial circuit, and their uses are widespread by the mass media. Other plants and products remain restricted to immigrant segments or family traditions, and they are invisible for the majority of the local population. Nevertheless, some of these invisible vegetal elements become visible when they enter the general commercial circuit. In terms of the UBK dynamics, a restricted component (linked to traditions) spreads, and it gets generalized through a visualization process, in which the mass media play a relevant role (Hurrell et al. 2011a; Pochettino et al. 2012a).

\section{Study area/Involved actors}

The conurbation Buenos Aires-La Plata includes the Greater Buenos Aires, an urban agglomeration emerged around Buenos Aires city (Federal District) and the Greater La Plata, a contiguous agglomeration emerged around La Plata city (Figure 1). Greater Buenos Aires comprise the Federal District and 24 districts of the Buenos Aires province. Its total area is $3,833 \mathrm{~km}^{2}$ (AABA 2013; Gemini 2003). Buenos Aires city has 2,891,082 inhabitants (in an area of $202 \mathrm{~km}^{2}$ ), while the 24 districts of Buenos Aires province have a total of 9,910,282 inhabitants (INDEC, 2013). In population, Greater Buenos Aires is the largest agglomeration in Argentina, the second in South America (after the metropolitan area of São Paulo, Brazil), the third in Latin America (after the metropolitan areas of Mexico and São Paulo), the fifth of America and the seventeenth worldwide (Forstall et al. 2004). The Greater La Plata is an urban agglomeration constituted by three districts of the Buenos Aires province: La Plata (which includes La Plata city), Berisso and Ensenada. Its total area is of $1,162 \mathrm{~km}^{2}$, and its population of 793,365 inhabitants (INDEC 2013).

This conurbation is heterogeneous, and it comprises at least three different spaces: 1. Urban areas themselves; 2 . Non-urbanized areas with native vegetation (some sectors correspond to protected natural areas); 3. Periurban areas considered transitional zones between urban and non-urbanized areas, characterized by moving boundaries which fluctuate according to the rhythms of urbanization. In the periurban area an intense horticultural activity is developed, whose production supplies fresh vegetables and fruits to the inhabitants of the conurbation and other urban areas of the country (Barsky 2005, 2010; Hurrell et al. 2011b). The set of homegardens and productive orchards is called horticultural belt (Pochettino et al. 2012b). 


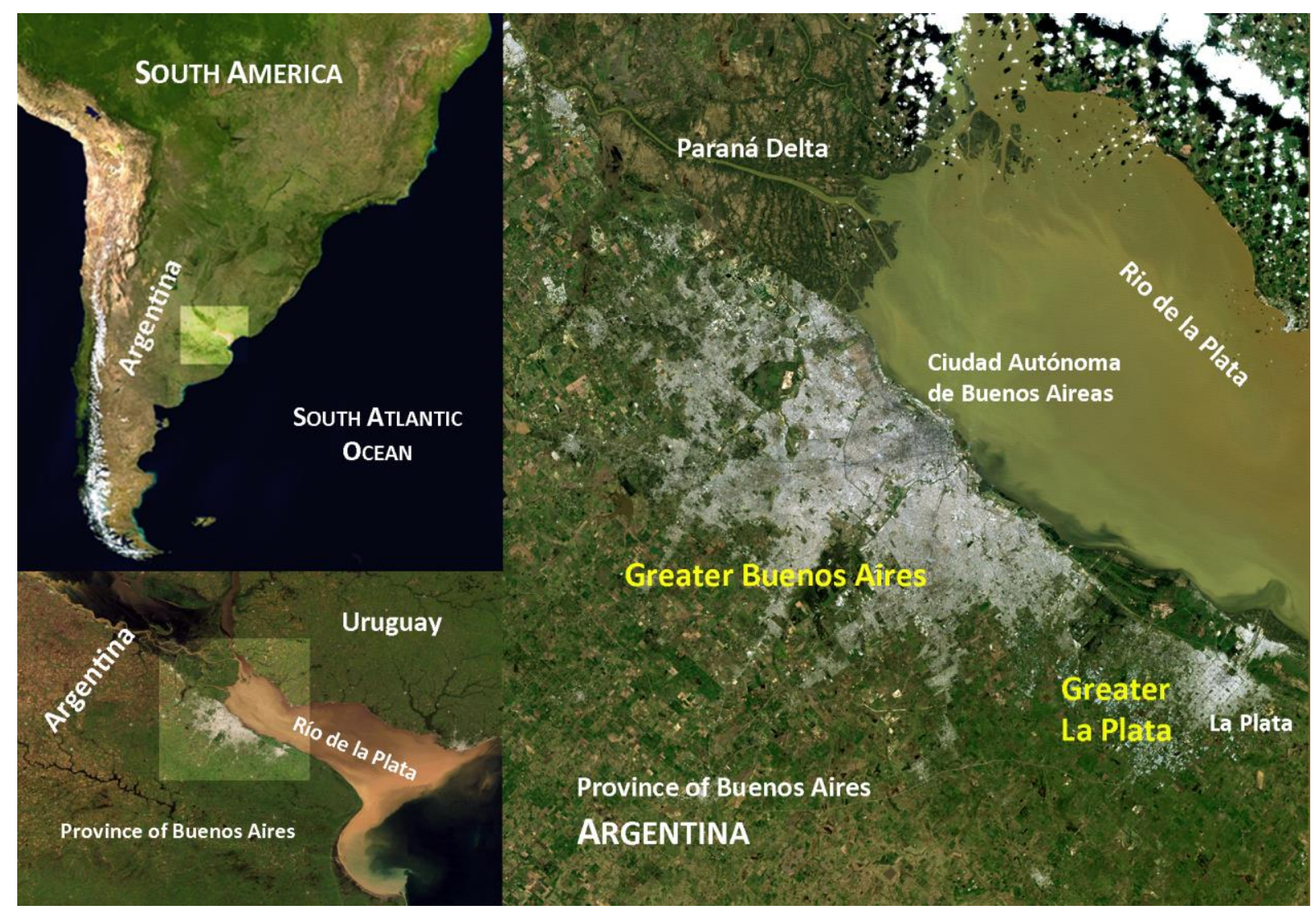

Figure 1. Conurbation Buenos Aires-La Plata, comprising the urban agglomerations of Greater Buenos Aires and Greater La Plata (satellite images from NASA).

Argentina has received massive immigration waves from the mid nineteenth century and first half of the twentieth century. Most of these immigrants were of European origin: $44.9 \%$ Italian and $31.5 \%$ Spaniards from the total of immigrants registered until 1940 (Cerrutti 2009). These migration flows have helped to shape the country's cultural heritage, and many current 'family traditions' have their roots in that early immigration. In the second half of the twentieth century was occurred a new recent immigration. It was not massive, and focused in the metropolitan area. These immigrants were oriented towards horticultural practices in periurban areas, as well as towards manufacturing industry, construction and commerce in urban areas themselves. Most of this immigration comes from Paraguay and Bolivia (respectively, $21.22 \%$ and $15.24 \%$ from all foreigners in 2001). Bolivian immigrants are an example of the pattern that connects the periurban areas (production) with the strictly urban areas (consumption). Immigrants dedicated to horticulture provide food for the urban sector, where other Bolivian immigrants commercialize the products: eg, at Bolivian traditional market of Liniers, a neighborhood of Buenos Aires city (for a specific description of this traditional market nestled in a characteristic urban scenario, see Pochettino et al. 2012a).

Another recent immigration into the conurbation corresponds to Far Eastern countries (Japan, Korea, and China). Asian immigration in 2001 represented almost $2 \%$ of all foreigners in the country, meager value compared to $67.96 \%$ coming from American countries and $28.22 \%$ from European countries (INDEC 2013). In the first half of the twentieth century Chinese immigration was low and settled in periurban areas, dedicated to horticulture (such as Bolivian immigrants). In the late twentieth century, these immigrants exceeded in number the Japanese and the Koreans ones, 
who previously dominated in the urban sector. At present, the Chinese community in Buenos Aires city has several restaurants, shops and supermarkets concentrated in the named Barrio Chino, a sector of neighborhood of Belgrano (Bogado Bordazar 2003). The Chinese supermarkets constitute a center for disseminating its BK linked to their traditions towards the local urban residents. Barrio Chino is not a traditional market, such as the Bolivian traditional market of Liniers, but it acts as a relevant visualization agent for the UBK dynamics comprehension.

\section{Materials and Methods}

The Bolivian and Chinese immigrants were considered as reference groups for the study of UBK linked to traditions. This knowledge can be extrapolated from the analysis of plants or plant products circulating at the traditional market of Liniers (named Bolivian market for the people that does not belong to the immigrant segment), and at Barrio Chino supermarkets of Belgrano. Exclusive elements at those markets indicate that are invisible for most of the conurbation inhabitants. On the contrary, plants or plant products found in general commercial circuit stores, or sold via Internet, are visible elements that are linked to nontraditional UBK. Also, if an element is found in those reference markets, and also in general commercial circuit, although less frequently, it is considered as an element in process of visualization. From these distinctions, the information about plants and/or their derived products was evaluated.

The ethnobotanical data collection followed the usual qualitative techniques and methods (Albuquerque and Lucena 2004; Alexíades and Sheldon 1996; Martin 2004), such as participant observation, free listings, open-ended and semi-structured interviews. For the Bolivian market, criteria were adjusted according to the studies in traditional markets (Cunningham 2001; Macía et al. 2005).

The partial results presented here correspond to the plant family Asteraceae, comprising numerous species (mostly contain essential oils) used as therapeutic and aromatic (specially as a food and beverage flavoring). In all cases, samples were obtained from plants and their products, which were deposited in the LEBA, and herbarium specimens are found in the Herbarium of Museo de La Plata (LP). The samples were examined according to their characteristics. Plants and parts thereof (such as roots and seeds) were identified from external morphological traits. The dry materials, fragmented or powdered, were identified through the micrographic analysis of external and internal morphological features. From this analysis, possible adulterations will be shortly evaluated.

The field surveys were conducted in diverse outlets of plant products. The procedures always were performed with the consent of the informants. The sellers have been considered as 'qualified informants', they are people of both sexes and different ages that demonstrated their knowledge about the properties of the plant products they sell. All of them showed a positive attitude to provide the requested data. In Bolivian market, 30 premises and street stalls (all the outlets) have been visited and 50 sellers have been interviewed. Up to now, the studies in Barrio Chino were developed into the 3 major supermarkets, with 12 sellers interviewed. For the general commercial circuit, the main outlets surveyed were the health-food stores, locally called dietéticas: 52 of them were relieved and 100 sellers were consulted. In total, 58 outlets surveyed and 162 qualified informants interviewed.

The dietéticas are places that concentrate the interest on plants that contribute to health, and are privileged sites chosen by the local people for the supply of healthy food, dietary supplements, mother tinctures, and herbal products for therapeutic 
purposes. Decades ago, the sale of herbal products was the patrimony of herbalist shops (called herboristerías), but at present these places are almost extinguished, and herbal products are usually sold in dietéticas (also in some pharmacies). These shops are furthermore true dispersal centers for plant products (expansion that also enhanced by the mass media). In the surveyed dietéticas, the presence of different products coming from the restricted frame of the immigrant segments, both Bolivian and Chinese, has been confirmed (Arenas et al. 2011; Hurrell et al. 2013). Once in the dietéticas, those invisible products gain the general commercial circuit and become visible. In this sense, the dietéticas are true visualization agents.

The available literature about the observed species and their uses was revised (in particular: Alonso and Desmarchelier 2005; Barboza et al. 2009; Burgstaller 1968; Freire and Urtubey 1999a,b, 2000a,b,c; Hieronymus 1882; Martínez Crovetto, 1981; Sorarú and Bandoni 1978; Zardini 1984a,b). Also, the information from printed and electronic labels, leaflets and advertisements belonging to many products was evaluated (because it guides people in the selection of products to be consumed). Both the data of these additional sources as those from the literature, together with the obtained in the field work, provide the repertoire of uses assigned for the species under study. Once identified these uses, the biological activity and effects registered in different investigations were explored, in order to make comparisons.

\section{Results and Discussion}

Table 1 includes information about 50 species of Asteraceae commercialized as medicinal and aromatic in the study area. Each species is indicated by it scientific name (in alphabetical order). In the first column, its local names, geographical origin, type of products, and samples obtained were also included. The products comprise: dietary supplements (DS), essential oil (EO), fresh plants (FP), fresh roots (FR), herbal products (HP), mother tinctures (MT), ointment (OI); powdered materials (PW), seeds (SE), tea bags (TB) and tea bags with mixture of herbs for medicinal infusions (TM). Second column contains the uses reported from interviews, the data from labels, leaflets, advertisements, catalogs, and the available literature. Besides medicinal $(M)$ and aromatic $(A)$ uses, other recognized local uses are added (O). Third column includes the therapeutic effects and biological activity registered in scientific investigations (the bibliographic sources are indicated in each case). The particular information for each species that emerges from the table is complemented by the main following remarks to get an overview.

Species/Products: The variety of observed products shows the diversity of forms of consumption. For some species also shows their high degree of diffusion and uses (visibility), eg Matricaria chamomilla, Smallanthus sonchifolius, Stevia rebaudiana. Herbal products are most widespread vegetal elements (43 species: $86 \%$ of the total), mainly employed to prepare therapeutic infusions. The abundance of these products also is justified because is the cheapest sold material. Herbals are followed by mother tinctures $(50 \%$ of the species), likewise used for medicinal reasons. Those tinctures are more expensive than herbal products, but its consumption is easier. The ease in the form of consumption is an important selection criterion for many people when selecting what product is to be used. The same applies for dietary supplements (10\% of the species), even more expensive but easier to consume (this product is the most widespread by the massive media). One species is only marketed as a dietary supplement, Hieracium pilosella L., however its diffusion is wide because it is sold mostly through the Internet. 
Table 1. Medicinal and aromatic Asteraceae commercialized in the study area.

\begin{tabular}{l}
\hline SCIENTIFIC/LOCAL NAMES. \\
GEOGRAPHICAL ORIGIN \\
PRODUCTS [SAMPLES] \\
\hline Acanthospermum \\
australe (Loefl.) Kuntze \\
TAPECUÉ \\
America \\
HP [H218] \\
OI [P159 $\left.\left(^{*}\right)\right]$
\end{tabular}

\begin{tabular}{l}
\hline Acanthostyles buniifolius \\
(Hook. ex Arn.) R.M. King \\
\& H. Rob. [= Eupatorium \\
buniifolium Hook. \& Arn.] \\
RomeriLLO
\end{tabular}

Southern South America

HP [H201]

MT [H349]

Achillea millefolium L

MiLENRAMA-AQUILEA

Europe and Western Asia

HP [C001]

MT [H348]

\section{USES REPORTED (INTERVIEWS, LEAFLETS, LABELS, ADVERTISEMENTS, CATALOGS, LITERATURE) \\ BIOLOGICAL ACTIVITIES AND EFFECTS RECORDED (BIBLIOGRAPHICAL REFERENCES)}

M: Hepatic, astringent, antidiarrheal, antiulcerative, antitumor, diuretic, antiinflammatory, antirheumatic, antiarthritic, febrifuge, antimalarial, sudorific, depurative, sedative, hypnotic, analgesic, antidermatitis, vulnerary, antiseptic, antigonorrheal, contraceptive, abortifacient.

M: Hepatic, choleretic, digestive, tonic, antirheumatic, antinephritic, anti-inflammatory, analgesic, sedative, hypnoptic, antiseptic.

$\mathbf{O}$ : Tinctorial, insect repellent.

M: Astringent, antispasmodic, carminative, digestive, anthelmintic,

laxative, antidiarrheal, cholagogue, choleretic, hepatic, antihaemorrhoidal, diuretic, antinephritic, hypotensive, sedative, anxiolytic, emmenagogue, febrifuge, expectorant, anti-influenza, anti-inflammatory, antirheumatic, antiseptic, vulnerary.

[Matricaria chamomilla L. adulterant]. A: Beverage flavoring, perfumery. O: Ornamental, edible (leaves).

Achyrocline satureoides (Lam.) DC.

MARCELA

Southern South America HP [C020] [C139] [H097]

MT [H345]

TM [H412]
M: Hepatic, carminative, stomachic, tonic, digestive, antispasmodic, antiulcerative, antidiarrheal, anthelmintic, stimulant, febrifuge, antidiabetic, emmenagogue, sedative, antitumor, expectorant, antitussive, antiasthmatic, hypocholesterolemic, anti-inflammatory, analgesic, antiseptic, vulnerary, slimming.

A: Beverage flavoring.

O: Insect repellent.
Antitumor (Mirandola et al. 2002), antiviral (Rocha Martins et al. 2011), antifungal (Portillo et al. 2001), antimalarial (Carvalho and Krettli 1991), antioxidant (Desmarchelier et al. 1994), inhibiting fertilization (Barboza et al. 2009). Immunomodulatory (Fernández et al. 2002), antioxidant (Soria et al., 2008), antimicrobial (Muschietti et al. 2005; Sülsen et al. 2007), antiviral (HIV) (Hnatyszyn et al. 1999), antiinflammatory (Muschietti et al. 2001), antinociceptive (Miño et al. 2005), hypnoptic, amnesic (Miño et al. 2007). Antitumor (Csupor-Löffler et al. 2009), cytoprotective, anti-inflammatory (Zaidi et al. 2012), analgesic (Pires et al.

2009), antioxidant, antimicrobial (Falconieri et al. 2011; Vitalini et al. 2011), hypotensive (Souza et al. 2011), vasoprotective (Dall'Acqua et al. 2011), bronchodilatory (Khan and Gilani 2011), antispasmodic and hepatoprotective (Yaeesh et al. 2006), choleretic (Benedek et al. 2006), anxiolytic (Baretta et al. 2012). Cytotoxic (Ruffa et al. 2002), immunomodulatory (Cosentino et al. 2008), antimicrobial (Casero et al. 2013), antiviral (Sabini et al. 2012), anti-inflammatory (Barioni et al. 2013), analgesic (Rondina et al. 2008), antioxidant (Gugliucci and Menini 2002), hypoglycemic (Heng et al. 2010), hypocholesterolemic (Espiña et al. 2012), antiulcerative (Santin et al. 2010), muscle relaxant (Hnatyszyn et al. 2004), hepatoprotective (Kadarian et al. 2002), antispasmodic and hypotensive (Petenatti et al. 2004b). Antitumor (Spjut 2005), cytotoxic, analgesic, anti-inflammatory (Yukes \& Balick 2010), antiallergic, immunomodulatory (Broide 2009), antimicrobial (Chalchat et al. 2004), contraceptive (Mats et al. 1987).
Ambrosia elatior $\mathrm{L}$. [=A. artemisiifolia L.] Altamisa America HP [P149]
M: Anthelmintic, purgative, emetic, antispasmodic, stomachic, digestive, appetizer, carminative, astringent, antidiarrheal, choleretic, antitussive, febrifuge, antirheumatic, antiarthritic, antidote, antiallergic, analgesic, antineuralgic, anticephalalgic, antinephritic, antilithic, stimulant, emollient, antitumor, antidermatitis, vulnerary, antiseptic, emmenagogue, postpartum recovery, contraceptive, abortifacient.

O: Oil (seeds), insecticide. 


\begin{tabular}{|c|c|c|}
\hline $\begin{array}{l}\text { Arctium lappa L. } \\
\text { BARDANA } \\
\text { Eurasia } \\
\text { HP }[\mathrm{H} 282][\mathrm{H} 363] \\
\text { MT }[\mathrm{H} 352] \\
\left.\text { FR }\left[\mathrm{H} 6880{ }^{(* *}\right)\right]\end{array}$ & $\begin{array}{l}\text { M: Diuretic, antinephritic, antilithic, } \\
\text { hepatic, cholagogue, choleretic, } \\
\text { digestive, antispasmodic, astringent, } \\
\text { antidiabetic, anti-inflammatory, } \\
\text { antiulcerative, antitumor, depurative, } \\
\text { vulnerary, antidermatitis, antiaging, } \\
\text { antiseborrheic, antialopecic. } \\
\text { O: Edible (roots). }\end{array}$ & $\begin{array}{l}\text { Antitumor (Machado et al. 2012), } \\
\text { antioxidant (Song et al. 2010), anti- } \\
\text { inflammatory (Lee et al. 2012), } \\
\text { antiulcerative (Silva et al. 2013), } \\
\text { gastroprotective (Dos Santos et al. } \\
\text { 2008), hypoglycemic (Chan et al. } \\
\text { 2011), antispasmodic (Almeida et al. } \\
\text { 2013), antiviral (Hayashi et al. 2010). }\end{array}$ \\
\hline $\begin{array}{l}\text { Arnica montana L. } \\
\text { ÁRNICA } \\
\text { Europe } \\
\text { HP [H141] } \\
\text { MT [H355] }\end{array}$ & $\begin{array}{l}\text { M: Vulnerary, antidermatitis, anti- } \\
\text { inflammatory, analgesic, antiarthritic, } \\
\text { antirheumatic, cardiotonic, antiseptic, } \\
\text { immunostimulant, astringent, } \\
\text { antispasmodic. } \\
\text { A: Beverage flavoring, perfumery. }\end{array}$ & $\begin{array}{l}\text { Antitumor, antimicrobial, analgesic, } \\
\text { anti-inflammatory, antihistamine } \\
\text { (Vanaclocha and Cañigueral 2003; } \\
\text { Stanciuc et al. 2011), antioxidant } \\
\text { (Craciunescu et al. 2012), antiarthritic } \\
\text { (Widrig et al. 2007). }\end{array}$ \\
\hline $\begin{array}{l}\text { Artemisia absinthium L. } \\
\text { AJENJO } \\
\text { Eurasia and Northen } \\
\text { Africa } \\
\text { HP [C005] [P144] } \\
\text { MT [H356] } \\
\text { FP }\left[\mathrm{H} 165\left(^{\star}\right)\right]\end{array}$ & $\begin{array}{l}\text { M: Anthelmintic, antispasmodic, } \\
\text { appetizer, digestive, stomachic, } \\
\text { carminative, antiemetic, cholagogue, } \\
\text { hepatic, tonic, antimalarial, febrifuge, } \\
\text { diuretic, cardiotonic, hypotensive, } \\
\text { hypocholesterolemic, antirheumatic, } \\
\text { emmenagogue, mnemonic, hypnotic, } \\
\text { antiseptic, aphrodisiac, abortifacient. } \\
\text { A: Beverage flavoring. } \\
\text { O: Insecticide, insect repellent. }\end{array}$ & $\begin{array}{l}\text { Antitumor, cytotoxic (Shafi et al. 2012; } \\
\text { Wegiera et al. 2012), antimicrobial } \\
\text { (Stanciuc et al. 2011), antimalarial } \\
\text { (Irshad et al. 2011), anthelmintic (Tariq } \\
\text { et al. 2009), hepatoprotective (Amat et } \\
\text { al. 2010), antioxidant (Craciunescu et } \\
\text { al. 2012), cognitive desorders (Howes } \\
\text { et al. 2003), neuroprotective (Bora and } \\
\text { Sharma 2010). }\end{array}$ \\
\hline $\begin{array}{l}\text { Artemisia dracunculus L. } \\
\text { EsTRAGóN } \\
\text { North America and } \\
\text { Eurasia } \\
\text { HP [C025] [C103] } \\
\text { PW [C117] }\end{array}$ & $\begin{array}{l}\text { M: Hepatic, stomachic, carminative, } \\
\text { appetizer, anthelmintic, antiscorbutic, } \\
\text { antidiabetic, diuretic, emmenagogue, } \\
\text { anti-inflammatory, antigout, hypnotic, } \\
\text { antiepileptic. } \\
\text { A: Condiment, beverage flavoring, } \\
\text { perfumery. }\end{array}$ & $\begin{array}{l}\text { Antimicrobial, antioxidant (Benli et al. } \\
\text { 2007; Lopes-Lutz et al. 2008), } \\
\text { hepatoprotective, anti-inflammatory } \\
\text { (Obolskiy et al. 2011), hypoglycemic } \\
\text { (Ribnicky et al. 2006), antiplatelet } \\
\text { (Tognolini et al. 2006), anticonvulsant } \\
\text { (Sayyah et al. 2004). }\end{array}$ \\
\hline $\begin{array}{l}\text { Artemisia vulgaris L. } \\
\text { ARTEMISA } \\
\text { Eurasia and Northen } \\
\text { Africa } \\
\text { HP [C089] [H072] } \\
\text { MT [H347] }\end{array}$ & $\begin{array}{l}\text { M: Antispasmodic, carminative, } \\
\text { appetizer, digestive, anthelmintic, } \\
\text { cholagogue, choleretic, diuretic, } \\
\text { febrifuge, expectorant, antiasthmatic, } \\
\text { astringent, emmenagogue, antiseptic, } \\
\text { tonic, anticonvulsant, antidepressant. } \\
\text { A: Condiment, beverage flavoring. } \\
\text { O: Insecticide, insect repellent. }\end{array}$ & $\begin{array}{l}\text { Antitumor (Abdelhamed et al. 2013), } \\
\text { antimicrobial (Poiată et al. 2009), } \\
\text { antiviral (Meneses et al. 2009), } \\
\text { antispasmodic, bronchodilatory (Khan } \\
\text { and Gilani 2009), antioxidant (Temraz } \\
\text { and El-Tantawy 2008), anti- } \\
\text { inflammatory (Tigno and Gumila } \\
\text { 2000), analgesic (Pires et al. 2009). }\end{array}$ \\
\hline $\begin{array}{l}\text { Baccharis articulata } \\
\text { (Lam.) Pers. } \\
\text { CARQUEJA } \\
\text { Southern South America } \\
\text { HP [P143] } \\
\text { MT [H346] } \\
\text { FP [B416(*)] }\end{array}$ & $\begin{array}{l}\text { M: Digestive, hepatic, cholagogue, } \\
\text { antispasmodic, hypocholesterolemic, } \\
\text { antidiarrheal, anthelmintic, laxative, } \\
\text { diuretic, febrifuge, tonic, cardiotonic, } \\
\text { depurative, antidiabetic, against } \\
\text { urinary and respiratory infections, } \\
\text { antirheumatic, vulnerary, antiseptic, } \\
\text { antiulcerative, against male impotence } \\
\text { and female infertility; aphrodisiac, } \\
\text { contraceptive. } \\
\text { A: Beverage flavoring. }\end{array}$ & $\begin{array}{l}\text { Antioxidant (Verdi et al. 2005), } \\
\text { antimicrobial (Simoniato et al. 2008), } \\
\text { antiviral (Torres et al. 2011), anti- } \\
\text { inflammatory (Gené et al. 1992), } \\
\text { hypoglycemic (Kappel et al. 2012). }\end{array}$ \\
\hline $\begin{array}{l}\text { Baccharis crispa Spreng. } \\
\text { CARQUEJA- CARQUEJA } \\
\text { CRESPA } \\
\text { Southern South America } \\
\text { HP [H362] } \\
\text { MT [H342] }\end{array}$ & $\begin{array}{l}\text { M: Digestive, hepatic, cholagogue, } \\
\text { anti-icteric, antilithic, antispasmodic, } \\
\text { anthelmintic, tonic, antiasthmatic, } \\
\text { antidiabetic, diuretic, antirheumatic, } \\
\text { depurative, febrifuge, aphrodisiac, } \\
\text { against male impotence and female } \\
\text { infertility, antiulcerative, antiseptic, } \\
\text { vulnerary, antiacne, antiseborrheic, } \\
\text { antidandruff. } \\
\text { A: Beverage flavoring. }\end{array}$ & $\begin{array}{l}\text { Antioxidant, antimicrobial (Verdi et al. } \\
\text { 2005), antiviral (Mangiaterra 2005), } \\
\text { anti-inflammatory (Gené et al. 1992). }\end{array}$ \\
\hline
\end{tabular}




Baccharis salicifolia (Ruiz
\& Pav.) Pers.
[= B. glutinosa Pers.]
CHILCA
America
HP $[\mathrm{H} 416]$

Baccharis trimera (Less.)

DC.

Carqueja-Carquejilla

Southern South America

HP [C017]

FP $\left[\mathrm{B} 424\left(^{*}\right)\right]$
M: Anti-inflammatory, antirheumatic, analgesic, astringent, antidiarrheal, antidysenteric, antiulcerative, antiacid, digestive, hepatic, anthelmintic, antiseptic, antisyphilitic, against gynecological disorders, diuretic, antitumor, febrifuge, antialopecic, antidermatitis.

O: Insecticide, insect repellent.

M: Digestive, hepatic, cholagogue, antispasmodic, anthelmintic, tonic, antiulcerative, febrifuge, antidiabetic, diuretic, antirheumatic, analgesic, anti-inflammatory, hypotensive, antiseptic, vulnerary, antinephritic, aphrodisiac, against male impotence and female infertility, emmenagogue, against birth and postpartum ailments, neonatal care, abortifacient .

[Baccharis articulata (Lam.) Pers. and

$B$. crispa Spreng. substitute or adulterant].

Calendula officinalis L.
CALÉNDULA
Europe
HP [C160] [H200]
MT [H341]

M: Emollient, antidermatitis, antiinflammatory, antiseptic, vulnerary, digestive, antispasmodic, hepatic, cholagogue, choleretic, astringent, antihaemorrhoidal, febrifuge, antiatherosclerotic, emmenagogue, hypotensive, depurative.
A: Perfumery.
O: Ornamental, cosmetics, edible (flowers).

Chamaemelum nobile (L.) M: Sedative, anxiolytic, digestive, All. [= Anthemis nobilis L.] stomachic, carminative, hepatic, MANZANILLA ROMANA Eastern Europe and Northwestern Africa EO [H440] antispasmodic, antiulcerative, antiacid, anthelmintic, appetizer, laxative, antiophthalmic, antiotitis, tonic, antirheumatic, anti-inflammatory emmenagogue, hypotensive, antiseptic, antidermatitis, vulnerary. [Matricaria chamomilla L. adulterant]. A: Food and beverage flavoring. O: Cosmetics, insect repellent. Chrysanthemum M: Hepatic, carminative, febrifuge, morifolium Ramat. [=C. grandiflorum (Desf.) Dum. Cours.] Crisantemo Eastern Asia HP [H420] anticephalalgic, expectorant, anticold, depurative, antiphlebitis, antiatherosclerotic, anti-inflammatory, antitumor, sedative, hypnotic, antiophthalmic, antiseptic, vulnerary. A: Food and beverage flavoring. O: Ornamental. Edible (leaves, flowers).

\section{Cichorium intybus $\mathrm{L}$.}

ACHICORIA

Eurasia and Northern

Africa

HP [H129]

TM [H410] M: Hepatic, cholagogue, appetizer, digestive, laxative, antidiarrheal, anthelmintic, anti-icteric, depurative, hipotensive, antimalarial, antidiabetic, anti-inflammatory, antiasthmatic, antisyphilitic, diuretic, antirheumatic, antiophthalmic, vulnerary, slimming. O: Edible (roots, leaves, flowers).
Anti-inflammatory (Gonzales Dávalos et al. 2007), antimicrobial (Verástegui et al. 1996), anthelmintic (Salazar et al. 2007).

Antioxidant (Pádua et al. 2010), hepatoprotective, antimutagenic (Verdi et al., 2005), hypoglycemic (Oliveira et al. 2005), antiparasitic (Gianello et al. 2000), immunomodulatory (Paul et al. 2009), antimicrobial, antiviral

(Mangiaterra 2005), analgesic, antiinflammatory (Gené et al. 1992, 1996), vasodilatory (Hnatyszyn et al. 2003). Antitumor (Matić et al. 2012; Wegiera et al. 2012), antimicrobial (Szakiel et al. 2008), antiviral (HIV) (Kalvatchev et al. 1997), antidermatitis (Fonseca et al. 2010), anti-inflammatory (Preethi et al. 2009), hepatoprotective, nephroprotective (Preethi and Kuttan 2009), anti-atherosclerotic (Orekhov 2013), antioxidant (Butnariu and Coradini 2012).

Antitumor, antioxidant (Guimarães et al. 2013), antimicrobial (Bail et al. 2009; Duarte et al. 2005), hypoglycemic (König et al. 1998), vasorelaxant (Zeggwagh et al. 2013), anti-inflammatory, sedative (Rossi et al. 1988), anxiolytic (Setzer 2009). Antitumor (Xie et al. 2009), antiviral (HIV) (Lee et al. 2003), antimicrobial (Akihisa et al. 2005), anti-inflammatory (Ukiya et al. 2001), cardiovascular protective (Lii et al. 2010), antioxidant (Song et al. 2010), neuroprotective (Lin et al. 2010), hypnotic (Kim et al. 2011).

Antitumor (Conforti et al. 2008), antibacterial (Aqil and Ahmad 2007), hepatoprotective (Atta et al. 2010), antioxidant (Lavelli 2008), anthelmintic (Foster et al. 2011), anti-inflammatory (Minaiyan et al. 2012), hypoglycemic (Pushparaj et al. 2007), anti-obesity (Vasudeva et al. 2012). 


\begin{tabular}{|c|c|c|}
\hline $\begin{array}{l}\text { Cnicus benedictus L. } \\
\text { [= Centaurea benedicta } \\
\text { (L.) L.] } \\
\text { CARDO SANTO-CARDo } \\
\text { BENDITO } \\
\text { Mediterranean and Asia } \\
\text { Minor } \\
\text { HP [H202] } \\
\text { MT [H339] } \\
\text { TM [H413] }\end{array}$ & $\begin{array}{l}\text { M: Tonic, carminative, digestive, } \\
\text { stomachic, appetizer, emetic, hepatic, } \\
\text { cholagogue, astringent, antidiarrheal, } \\
\text { diuretic, hypoglycemic, depurative, } \\
\text { emmenagogue, anti-inflammatory, } \\
\text { antiarthritic, expectorant, febrifuge, } \\
\text { antimalarial, antitumor, galactogogue, } \\
\text { antiseptic, vulnerary, antidermatitis, } \\
\text { contraceptive, abortifacient. } \\
\text { A: Condiment, beverage flavoring. } \\
\text { O: Oil (seeds), food (inflorescences). }\end{array}$ & $\begin{array}{l}\text { Antitumor, cytotoxic (Steenkamp and } \\
\text { Gouws 2006; Tamayo et al. 2000), } \\
\text { antimicrobial (Voon et al. 2012), anti- } \\
\text { inflammatory (Mascolo et al. 1987), } \\
\text { galactogogue (Westfall 2003). }\end{array}$ \\
\hline $\begin{array}{l}\text { Conyza bonariensis (L.) } \\
\text { Cronquist } \\
\text { YERBA CARNICERA } \\
\text { South America } \\
\text { HP [H053] } \\
\text { MT [H359] }\end{array}$ & $\begin{array}{l}\text { M: Diuretic, antinephritic, hepatic, } \\
\text { stomachic, antiacid, antiulcerative, } \\
\text { anthelmintic, astringent, antidiarrheal, } \\
\text { antihaemorrhoidal, cardiotonic, } \\
\text { febrifuge, expectorant, antitussive, } \\
\text { antirheumatic, antigout, analgesic, } \\
\text { anti-inflammatory, anticephalalgic, } \\
\text { antidermatitis, antiseptic, vulnerary. }\end{array}$ & $\begin{array}{l}\text { Cytotoxic (El Zalabani et al. 2012), } \\
\text { antimicrobial (Souza et al. 2004), } \\
\text { antiviral (Wachsman et al. 1988), anti- } \\
\text { inflammatory (Souza et al. 2003), CNS } \\
\text { depressant, cardiotonic (Barboza et al. } \\
\text { 2009), antioxidant (Shahwar et al. } \\
\text { 2012), anticonstipation, antidiarrheal } \\
\text { (Bukhari et al., 2013). }\end{array}$ \\
\hline $\begin{array}{l}\text { Cyclolepis genistoides } \\
\text { D.Don } \\
\text { PALO AZUL } \\
\text { Southern South America. } \\
\text { HP [H007] } \\
\text { MT [H358] }\end{array}$ & $\begin{array}{l}\text { M: Diuretic, antinephritic, anti- } \\
\text { inflammatory, antirheumatic, antigout, } \\
\text { analgesic, muscle relaxant, hepatic, } \\
\text { cholagogue, antispasmodic, } \\
\text { antitussive, depurative. }\end{array}$ & $\begin{array}{l}\text { Antitumor (Yasukawa 1991; Pisha et } \\
\text { al. 1995), diuretic, anti-inflammatory } \\
\text { (Sosa et al. 2007, 2011), analgesic } \\
\text { (Rondina et al. 2008). }\end{array}$ \\
\hline $\begin{array}{l}\text { Cynara cardunculus L. } \\
\text { [= C. scolymus L.] } \\
\text { ALCACHOFA } \\
\text { Mediterranean } \\
\text { HP [H069] } \\
\text { MT [H333] } \\
\text { TB [H093(*)] } \\
\text { TM [H411] } \\
\text { DS }[\mathrm{HO94}]\end{array}$ & $\begin{array}{l}\text { M: Hepatic, cholagogue, choleretic, } \\
\text { anti-icteric, antilithic, antispasmodic, } \\
\text { appetizer, digestive, stomachic, } \\
\text { antiacid, antirheumatic, diuretic, } \\
\text { antinephritic, hypocholesterolemic, } \\
\text { antidiabetic, depurative, hypotensive, } \\
\text { slimming, aphrodisiac. } \\
\text { A: Beverage flavoring. } \\
\text { O: Edible (leaves, inflorescences), } \\
\text { tinctorial. }\end{array}$ & $\begin{array}{l}\text { Antitumor (Conforti et al. 2008; Mileo } \\
\text { et al. 2012), antioxidant (Falleh et al. } \\
\text { 2008), antimicrobial (Zhu et al. 2004), } \\
\text { hypoglycemic (Fantini et al. 2011), } \\
\text { antispasmodic (Emendörfer et al. } \\
\text { 2005), anti-inflammatory (Kammoun et } \\
\text { al. 2010), nephroprotective (Turgut et } \\
\text { al. 2008), hepatoprotective (Metwally } \\
\text { et al. 2011), hypocholesterolemic } \\
\text { (Wider et al. 2009). }\end{array}$ \\
\hline $\begin{array}{l}\text { Echinacea purpurea (L.) } \\
\text { Moench } \\
\text { EcHINÁcEA } \\
\text { North America } \\
\text { HP [H229] [H280] } \\
\text { MT [H334] } \\
\text { DS [A-E1] }\end{array}$ & $\begin{array}{l}\text { M: Tonic, immunostimulant, antiseptic, } \\
\text { antidote, antiallergic, expectorant, } \\
\text { anti-influenza, anti-inflammatory, } \\
\text { antitumor, depurative, antidiarrheal, } \\
\text { antigonorrheal, skin care, vulnerary, } \\
\text { aphrodisiac. } \\
\text { O: Ornamental, cosmetics. }\end{array}$ & $\begin{array}{l}\text { Antitumor (Skaudickas et al. 2009; Tsai } \\
\text { et al. 2012), immunostimulant, } \\
\text { antioxidant (Hudson 2012; Mishima et } \\
\text { al. 2004), antimicrobial (Canlas et al. } \\
\text { 2010), antiviral (Pleschka et al. 2009), } \\
\text { anti-cold (Nahas and Balla 2011), anti- } \\
\text { inflammatory (Yu et al. 2013). }\end{array}$ \\
\hline $\begin{array}{l}\text { Flaveria bidentis (L.) } \\
\text { Kuntze } \\
\text { CoNTRAYERBA } \\
\text { Central and South } \\
\text { America } \\
\text { HP [H343] }\end{array}$ & $\begin{array}{l}\text { M: Antidote, febrifuge, antitussive, } \\
\text { expectorant, stimulant, tonic, sudorific, } \\
\text { diuretic, digestive, stomachic, laxative, } \\
\text { anthelmintic, emmenagogue, against } \\
\text { leukorrhea, vulnerary, antiseptic. } \\
\text { O: Tinctorial, insecticide. }\end{array}$ & $\begin{array}{l}\text { Anthelmintic (Pastor and Zelada } \\
\text { 2006), antibacterial (Bardón et al. } \\
\text { 2007), antiviral (Barboza et al. 2009), } \\
\text { antithrombotic, anticoagulant } \\
\text { (Guglielmone et al. 2012), antioxidant } \\
\text { (Dadé et al. 2009). }\end{array}$ \\
\hline $\begin{array}{l}\text { Gaillardia megapotamica } \\
\text { (Spreng.) Baker } \\
\text { TOPASAIRE } \\
\text { Southern South America } \\
\text { HP [H148] [P153] } \\
\text { MT [H351] }\end{array}$ & $\begin{array}{l}\text { M: Antialopecic, antiseborrheic, } \\
\text { antidandruff, antiseptic, analgesic, } \\
\text { antineuralgic, anticephalalgic, } \\
\text { digestive, stomachic, expectorant, } \\
\text { decongestive, anti-influenza, } \\
\text { antiasthmatic, hypotensive. }\end{array}$ & $\begin{array}{l}\text { Antitumor (Bongiovanni et al. 2006), } \\
\text { antimicrobial (Rosella et al. 2010), } \\
\text { antioxidant, gastroprotective (Barboza } \\
\text { et al. 2009; Bucciarelli and Skliar } \\
\text { 2007; Petenatti et al. 2004a), } \\
\text { analgesic (Rondina et al. 2008). }\end{array}$ \\
\hline $\begin{array}{l}\text { Gnaphalium } \\
\text { gaudichaudianum DC. } \\
\text { VIRA VIRA } \\
\text { Southern South America } \\
\text { HP [H250] }\end{array}$ & $\begin{array}{l}\text { M: Digestive, carminative, hepatic, } \\
\text { cholagogue, antispasmodic, anti- } \\
\text { inflammatory, depurative, antidote, } \\
\text { cardiotonic, diuretic, febrifuge, } \\
\text { expectorant, antitussive, vulnerary, } \\
\text { emmenagogue, anaphrodisiac. }\end{array}$ & $\begin{array}{l}\text { obial, antiviral, antispasmodic } \\
\text { ti et al. 2004b). }\end{array}$ \\
\hline
\end{tabular}




Haplopappus rigidus Phil.
BAlLA BIEN
Southern South America
HP [H096]
MT [H268]

Helianthus annuus L.

GIRASOL

North America

SE [H408] [H409]
M: Aphrodisiac, against male impotence, hepatic, cholagogue, choleretic, digestive, stomachic, carminative, astringent, antidiarrheal, antidysenteric, antiseptic, antitussive, expectorant, antinephritic, antilithic, antirheumatic, emmenagogue.

M: Slimming, tonic, antioxidant astringent, emollient, expectorant, antiasthmatic, febrifuge, antidiabetic, hypocholesterolemic, depurative, cardiotonic, hypotensive, stomachic, anthelmintic, cathartic, diuretic, antirheumatic, anti-inflammatory, immunostimulant, anticephalalgic, neuroprotective, antinephritic, analgesic, antiseptic, against urinary and respiratory infections, vulnerary, antidermatitis.

O: Ornamental, oil, edible (seeds).
Helichrysum italicum (Roth) G. Don

CURRY

Mediterranean

FP $\left[\mathrm{H} 439\left(^{*}\right)\right]$

M: Expectorant, antiasthmatic, antitussive, antispasmodic, digestive, choleretic, hepatic, astringent, diuretic, analgesic, antineuralgic, anticephalalgic, anti-inflammatory, antirheumatic, anticoagulant, sedative, antiallergic, antiseptic, antidermatitis, vulnerary.

A: Food flavoring, perfumery.

O: Ornamental, insecticide.

Hieracium pilosella $\mathrm{L}$. [= Pilosella officinarum F.W. Schultz \& Sch. Bip.] VELLOSILLA Europe DS [H364]

M: Diuretic, antinephritic, slimming, febrifuge, expectorant, antitussive, antiasthmatic, anti-influenza, antiinflammatory, stomachic, choleretic, cholagogue, astringent, depurative, cardiotonic, hypotensive, antihemorrhagic, emmenagogue, antiseptic, vulnerary.

Matricaria chamomilla L.
[= M. recutita L.]
MANZANILLA
Eurasia
HP [C008] [H089]
MT [H357]
TB [H016] [H361]
FP $\left[B 427\left(^{*}\right)\right]$

Sedative, anxiolytic, hypnotic, mnemonic, tonic, stimulant, digestive, carminative, antispasmodic, emetic, antidiarrheal, antiulcerative, anthelmintic, anti-inflammatory, analgesic, hypocholesterolemic, emollient, expectorant, antitussive, emmenagogue, antinephritic, hypotensive, cardiotonic, febrifugue, vulnerary, antidermatitis, slimming. [Adulterated with Anthemis cotula L., Chamaemelum nobile (L.) All., Cladanthus mixtum (L.) Chevall., Tanacetum parthenium (L.) Sch. Bip.] A: Beverage flavoring, perfumery. O: Cosmetics.

M: Diuretic, astringent, antidiabetic, against respiratory and intestinal disorders, expectorant, antitussive, sedative, febrifuge, antirheumatic, antiarthritic, antihydrophobic, antidote, antidermatitis, vulnerary.
Cytotoxic, antitumor (Morales et al. 2009), antimicrobial (Morales et al. 2003), vasodilatory (Hnatyszyn et al. 2003), antilithic, hepatoprotective, uterine relaxant (Alonso 2005).

Antitumor, immunomodulatory (Plohmann et al. 1997), cytotoxic (Bader et al. 1996), antioxidant, antimicrobial (Giada and Mancini-Filho 2009; Subashini and Rakshitha 2012), antiviral (Oliveira et al. 2009), antiinflammatory (Akihisa et al. 1996), analgesic (Rondina et al. 2008), antiasthmatic (Heo et al. 2008), hypolipidemic (Saini and Sharma 2011).

Cytotoxic, antimicrobial, antiseptic (Ríos 2008), antiviral (Nostro et al. 2003), anti-inflammatory, antioxidant (Bauer et al. 2010; Sala et al. 2002).

Antitumor (Gawronska-Grzywacz et al. 2011), anti-inflammatory (GawronskaGrzywacz and Krzaczek 2006), diuretic (Beaux et al. 1999), antimicrobial (Frey and Meyers 2010), antioxidant (Stanojević et al. 2009).

Antitumor (Srivastava and Gupta

2007), immunomodulatory (Ghonime et al. 2011), antibacterial (Shikov et al. 2008), antifungal (Jamalian et al. 2012), antioxidant, hypoglycemic (Cemek et al. 2008), neuroprotective (Ranpariya et al. 2011), anxiolytic, hypnotic (Sarris et al. 2011), antidepressant (Amsterdam et al. 2012), mnemonic (Adams et al. 2007), antiulcerative (Duarte et al. 2011), anti-inflammatory (Zaidi et al. 2012), nephroprotective (Salama 2012), antiallergic (Chandrashekhar et al. 2011), vulnerary (Nayak et al. 2007).

Cytotoxic (Barboza et al. 2009), analgesic (Rondina et al. 2008), antioxidant, antifungal (Alonso and Desmarchelier 2005).
Southern South America HP [H254]
Mikania periplocifolia

Hook. \& Arn.

$$
\text { HP [H254] }
$$




\section{Pluchea sagittalis (Lam.) Cabrera \\ LUCERA-YERBA LUCERA \\ Southern South America HP [C045]}

M: Tonic, appetizer, stomachic, carminative, laxative, anthelmintic, antispasmodic, hepatic, cholagogue, choleretic, antidiarrheal, febrifuge, antidysenteric, antitussive, diuretic, expectorant, sedative, antirheumatic, antiphlebitis, antigonorrheal, antiseptic, vulnerary, abortifacient.

A: Beverage flavoring.
Porophyllum ruderale

(Jacq.) Cass.

QUIRQUIÑA

Caribbean, Central and

South America

FP $\left[B 413\left(^{*}\right)\right]$

Schkuhria pinnata (Lam.)

Kuntze ex Thell.

Canchalagua

America

HP [H150]

M: Antispasmodic, digestive, antiinflammatory, antiophthalmic, depurative, hemostatic, sudorific, analgesic, antiseptic, vulnerary.

A: Condiment.

M: Slimming, diuretic, digestive, stomachic, hepatic, depurative, resolutive, against respiratory and urinary tract infections, antimalarial, antidiabetic, anti-inflammatory, antirheumatic, antiseptic, antiacne, antidermatitis.

O: Insect repellent.

Senecio eriophyton J.

Rémy

Chachacoma

Argentina, Chile

HP [P154] hepatic, antinephritic, hypotensive, cardiotonic, altitude sickness,
Antioxidant, antimicrobial (Souza et al. 2004), anti-inflammatory (Pérez-

García et al. 1996), antinociceptive, gastroprotective (Figueredo et al. 2011), analgesic (Rondina et al. 2008), vulnerary (Schmidt et al. 2009). Cytotoxic, antimicrobial (Takahashi et al. 2013), antispasmodic (Alves 1996), antinociceptive, anti-inflammatory (Lima et al. 2011; Souza et al. 2003).

Antitumor (Rodrigo et al. 2010), antiinflammatory, antifungal (Barboza et al. 2009), antibacterial, antiacne (Bussmann et al. 2008; Wagate et al. 2010), antimalarial (Muthaura et al. 2007), hypoglycemic (Deutschländer et al. 2009).

M: Tonic, expectorant, antibronchitis, antitussive, antiasthmatic, digestive, antiseptic, emmenagogue, sedative, hypnotic, against male impotence.

A: Food and beverage flavoring.

Senecio filaginoides DC. [= S. albicaulis Hook. \& Arn.]

MATA MORA- VIRA VIRA

South America

HP [P150]

M: Expectorant, anticatarrhal, antitussive, febrifuge, sudorific, emmenagogue, analgesic (bone pain), antiseptic, vulnerary.

A: Food flavoring.
Antimicrobial (Barboza et al. 2009), vasodilatory (Hnatyszyn et al. 2003). tonic, altitude sickness, analgesic
Senecio nutans Sch. Bip. [= S. graveolens Wedd.]

Chachacoma de LA PUNA

South America

HP [H230]

M: Febrifuge, expectorant, antitussive, antiasthmatic, antibronchitis, antiinfluenza, digestive, antispasmodic, (bone pain), hypotensive, cardiotonic sedative, emmenagogue, vulnerary.

A: Condiment, beverage flavoring.

Senecio subulatus $\mathrm{D}$. Don ex Hook. \& Arn. var. erectus Hook. \& Arn.

SALVIA DE LA PUNA

Argentina

HP [H134]

MT [H360]

Silybum marianum (L.)

Gaertn.

Cardo mariano

Mediterranean

SE [H154]

MT [H354]
M: Expectorant, against catarrh, chronic bronchitis, dyspnoea, influenza, antitussive, antiasthmatic, digestive, antispasmodic, astringent, antihemorrhoidal, cardiotonic, anticephalalgic, antiotitis, antisyphilitic.

M: Hepatic (cirrhosis due to chronic hepatitis and alcoholism), choleretic, cholagogue, antilithic, anti-icteric, antitumor, digestive, stimulant, antiinflammatory, tonic, astringent, antihaemorrhoidal, diuretic, antidote, expectorant, emmenagogue, galactogogue, vulnerary.

O: Oil (seeds), edible (roots, leaves, inflorescences)
Antitumor (Cheung et al. 2010), antiinflammatory (Sharifi et al. 2013), immunomodulatory, antioxidant (Das and Mukherjee 2012), antiviral (Wagoner et al. 2010), hypoglycemic (Zhan et al. 2011), galactogogue (Di Pierro et al. 2008), nephroprotective (Turgut et al. 2008), hepatoprotective, hypocholesterolemic (Krecman et al. 1998; Shaker et al. 2010). 


Smallanthus sonchifolius
(Poepp. \& Endl.) H. Rob.
YAcoN
South America
DS [H286] [H293]
HP $\left.\left[\mathrm{H} 3322^{(*}\right)\right]$
MT $[\mathrm{H} 285]$
FR $\left[\mathrm{H} 6891\left(^{*}\right)\right]$

Stevia rebaudiana

(Bertoni) Bertoni

Yerba Dulce-Stevia

Southern South America

DS [H116] [H118] [H317]

HP [H198] [P147]

MT [H350]

FP $\left[\mathrm{B} 415\left(^{*}\right)\right]$

\section{Tagetes minuta L. HuACATAY-SUICO America HP [H415] FP $\left[\mathrm{B} 403\left(^{*}\right)\right]$}

M: Antidiabetic, stimulant, tonic, antitumor, digestive, stomachic, antidiarrheal, anticonstipation, prebiotic, hypocholesterolemic, antioxidant, anti-atherosclerotic, slimming, diuretic, antinephritic, immunostimulant, hypotensive, improving vision, fortifying bones and teeth (Calcium assimilation), skin antiaging, vulnerary.

O: Edible (roots).

M: Antidiabetic, emollient, antitussive, appetizer, digestive, anticonstipation, laxative, antiacid, diuretic, cardiotonic, hypotensive, depurative, slimming, vulnerary, antiseptic, skin antiaging, antidermatitis, immunostimulant, anticaries, contraceptive.

O: Sweetener.
Antitumor (Moura et al. 2012), hypoglycemic (Ayvar et al. 2001), hypolipidemic (Habib et al. 2011), antioxidant (Aguilar and Bonilla 2009), antimicrobial (Choi et al. 2010), immunostimulant (Delgado et al. 2012), prebiotic (Pedreschi et al. 2003), hepatoprotective (Valentová et al. 2004), nephroprotective (Honoré et al. 2012), Calcium assimilation (Lobo et al. 2007).

Antitumor (Yasukawa et al., 2002), antioxidant (Shukla et al. 2009), hypoglycemic (Chen et al. 2005), hypotensive (Liu et al. 2003), nephroprotective (Shivanna et al. 2013), immunomodulatory, antiinflammatory (Boonkaewwan et al. 2006), antimicrobial (Takaki et al. 1985), antiviral (Oliveira et al. 2013), contraceptive (Gil et al. 2008).

M: Digestive, carminative, cathartic, antispasmodic, anthelmintic, diuretic, depurative, hypotensive, expectorant, antiasthmatic, against urinary and venereal infections, postpartum ailments, neonatal care, sedative, antidepressant, antidandruff, anti-tick, anti-pediculosis, antialopecic, antiinflammatory, analgesic, antitumor, aphrodisiac, antiabortifacient.

A: Condiment, beverage flavoring. O: Ornamental, tinctorial, insecticide.

Tanacetum parthenium

(L.) Sch. Bip.

SANTA MARía

Europe

HP [H223]

M: Febrifuge, anticephalalgic, antiinflammatory, antirheumatic, sedative, analgesic, antispasmodic, digestive, carminative, anthelmintic, antiseptic, hypotensive, emmenagogue. [Matricaria chamomilla $\mathrm{L}$. adulterant].

A: Condiment, beverage flavoring. O: Ornamental, insecticide.

Tanacetum vulgare $\mathrm{L}$. TANACETO

Eurasia HP [H214]

M: Anthelmintic, antispasmodic, tonic, digestive, carminative, febrifuge, hypotensive, diuretic, antinephritic, antirheumatic, anticephalalgic, antidermatitis, mnemonic, sedative, emmenagogue, abortifacient.

A: Food and beverage flavoring. O: Ornamental, insecticide. Antitumor (Ickes et al. 1973), antimicrobial (Al-Musayeib et al. 2012; Xu et al. 2012), antiviral (Ghaemi et al. 2004), antioxidant (Tereschuk et al. 1997), hypoglycemic (Ranilla et al. 2010), bronchodilatory, expectorant, spasmolytic, anti-inflammatory, hipotensive (Jawla et al. 2010), analgesic (Rondina et al. 2008), antidepressant (Martijena et al. 1998), anti-tick (Nchu et al. 2012).

Antitumor (Li et al. 2012; Mathema et al. 2012), antioxidant (Fischedick et al. 2012), antimicrobial (Polatoglu et al. 2010), cardiotonic, antispasmodic (Pareek et al. 2011), anti-inflammatory (Williams et al. 1999), anticephalalgic (Vanaclocha and Cañigueral 2003).

Antitumor (Spjut 2005), cytotoxic (Wegiera et al. 2012), antibacterial (Smirnova et al. 2012), antiviral (Álvarez et al. 2011), antioxidant (Juan-Badaturuge et al. 2009), immunomodulatory (Xie et al. 2007), hypotensive (Lahlou et al. 2008), antiinflammatory (Williams et al. 1999).

M: Hepatic, cholagogue, antilithic, astringent, laxative, febrifuge, antiicteric, antihaemorrhoidal, diuretic, antinephritic, expectorant, antitumor, antidiabetic, tonic, antidote, antiinflammatory, slimming, depurative, hypotensive, emmenagogue, antiophthalmic, antirheumatic, antiarthritic, antiseptic, antidermatitis. A: Food and beverage flavoring. O: Edible (leaves).
Antitumor, cytotoxic, antioxidant (Chun and Kitts 2003; Koo et al. 2004; Sigstedt et al. 2008), immunostimulant (Lee et al. 2012), antimicrobial (Yarnell and Abascal 2009), anti-inflammatory (Jeon et al. 2008), hypoglycemic (Önal et al. 2005), depurative (Modaresi and Resalatpour 2012), hepatoprotective (Mahesh et al. 2010), hypolipidemic (Choi et al. 2010). 


Tessaria integrifolia Ruiz
\& Pav.
PÁJARO BOBо
South America
HP [H227] [H335]
MT [H340]

M: Febrifuge, astringent, antitussive, antiasthmatic, expectorant, diuretic, antinephritic, hypocholesterolemic, hepatic, laxative, antiallergic, antiseptic (urinary infections), tonic, anti-inflammatory, antirheumatic, analgesic, antiodontalgic, depurative, against prostate and uterus ailments, antigonorrheal, vulnerary.

O: Paper pulp, timber, ashes for making soap.

Trixis divaricata (Kunth) Spreng. subsp. discolor (D.Don) Katinas [ $=T$. antimenorrhoea (Schrank) Kuntze var. discolor (D.Don) Cabrera] Contrayerba

South America HP [P146]

Tussilago farfara $\mathrm{L}$. TUSILAGO

Eurasia and Northern Africa HP [H215] hypocholesterolemic, vulnerary, antidermatitis, diuretic, antinephritic, antirheumatic, antiarthritic, against paralysis, antiophthalmic, antiantineuralgic, antiamenorrheic, emmenagogue, abortifacient.

O: Insecticide. illness, antiallergic, antispasmodic,
M: Antidote, febrifuge, sudorific, tonic, Antioxidant (Mayhua et al. 2012), depurative, antidiabetic, expectorant, antiulcerative (Pereira et al. 2005). antitussive, antiasthmatic, rubefacient, inflammatory, digestive, antiulcerative,

M: Emollient, expectorant, antitussive, Neuroprotective (Cho et al. 2005), antiasthmatic, anticatarrhal, against antioxidant (Song et al. 2010), colds, chronic bronchitis, emphysema, cardiovascular and respiratory chest complaints and other respiratory stimulant (Li and Wang 1988), astringent, antidiarrheal, stomachic, against gastric diseases, febrifuge, sudorific, depurative, caridotonic, sedative, antiphlebitis, antirheumatic, anti-inflammatory, stimulant, tonic, antiseptic, antidermatitis, vulnerary, abortifacient.

A: Food and beverage flavoring. O: Edible (leaves, inflorescences).

\section{Xanthium spinosum $\mathrm{L}$. \\ M: Diuretic, antinephritic, antilithic,}

Cepa caballo

Worldwide temperate zones

HP [H127]

MT [H344] antitussive, expectorant (Li et al. 2013), immunostimulant, antiinflammatory (Hwangbo et al. 2009), antimicrobial (Kokoska et al. 2002). digestive, stomachic, antispasmodic, hepatic, choleretic, cholagogue, laxative, emetic, antidiarrheal, antidysenteric, febrifuge, antimalarial, antiphlebitis, anti-atherosclerotic, hypocholesterolemic, depurative, resolutive, antirheumatic, antiinflammatory, antitussive, against measles, mumps, lung diseases and prostate ailments, antihydrophobic, antiophthalmic, anticephalalgic, sedative, analgesic, emollient, vulnerary, antidermatitis, antiseptic, antigonorrheal, contraceptive, abortifacient.

O: Tinctorial, coffee substitute (seeds).
Antimicrobial (Vivot et al. 2007), antiviral (Romio and Gurni 2007), antiinflammatory (Peluso et al. 1995), antioxidant (Ono et al. 2000).

\section{References:}

Products: DS, dietary supplement; EO, essential oil; FP, fresh plant; FR, fresh roots; HP, herbal product; MT, mother tincture; OI, ointment; PW: powdered material; SE, seeds; TB, tea bags; TM, tea bags with mixture of herbs for medicinal infusions. Uses: A, aromatic; M, medicinal; $\mathbf{O}$, others. Samples: $\left({ }^{*}\right)$ only in Bolivian traditional market; $\left({ }^{* *}\right)$ only in Chinese markets. 
Fresh plants or parts thereof (roots) are sold solely in the context of restricted circuit of immigrant segments: 9 species in the Bolivian market (indicated in Table 1 with ${ }^{*}$ ) and 1 species in a Barrio Chino supermarket (indicated in Table 1 with ${ }^{* *}$ ). The use of these species is related to their origin traditions, eg medicinal and edible roots of Smallanthus sonchifolius consumed in the Andean region, and those of Arctium lappa employed in China for the same purposes. The case of fresh plants of Helichrysum italicum for sale in the Bolivian market is peculiar. Informants report that it is used as a condiment and for medicinal purposes. It was the only place where these uses were registered. For study area, the plant is better known by the urban inhabitants as aromatic ornamental and it is cultivated in gardens.

The ointment of Acanthospermum australe and the tea bags of Cynara cardunculus are exclusive of the Bolivian market, imported from Bolivia. Regarding the last of these species, it is marketed in dietéticas as tea bags, but mixed with other herbs (not Asteraceae) for making hypocholesterolemic infusions. The tea bags are common in the dietéticas because consumers choose them for their easy mode of consumption. These products are highly visible within the nontraditional commercial circuit. The tea bags of Matricaria chamomilla are the most widely spread because its sedative effects. Other Asteraceae are found in tea bags with mixture of herbs for medicinal infusions: Achyrocline satureoides (digestive), Cnicus benedictus (against arthritis) and Cichorium intybus (hypotensive).

Fifteen species: Ambrosia elatior, Baccharis salicifolia, Chrysanthemum morifolium, Flaveria bidentis, Gnaphalium gaudichaudianum, Mikania periplocifolia, Pluchea sagittalis, Schkuhria pinnata, Senecio eriophyton, S. filaginoides, S. nutans, Tanacetum parthenium, T. vulgare, Trixis divaricata subsp. discolor, and Tussilago farfara, are only marketed as herbal products, and they are found only in a few dietéticas. Of these species, Chrysanthemum morifolium, Tanacetum parthenium, $T$. vulgare, and Tussilago farfara are not native to Argentina. The two Tanacetum species are growing naturalized in the country. Chrysanthemum morifolium is often cultivated as an ornamental. The herbal product of Tussilago farfara has been found only in one dietética, and it is imported. Chamaemelum nobile has only been found as essential oil (imported). As powder, only Artemisia dracunculus is expended (spice). From Silybum marianum, the seeds are mainly marketed (also as mother tincture). From Helianthus annuus, only its seeds are sold.

Of the 50 species surveyed, only 7 are included in the Farmacopea Argentina (the official Argentine pharmacopoeia): Baccharis articulata, B. trimera, Calendula officinalis, Cynara cardunculus, Matricaria chamomilla, Silybum marianum and Stevia rebaudiana. Other 3 species, Achyrocline satureoides, Arnica montana and Baccharis crispa, are recorded as phytomedicines by the ANMAT: Administración Nacional de Medicamentos, Alimentos y Tecnología Médica ('National Administration of Drugs, Food and Medical Technology'). Moreover, the agency referred considers toxic (prohibited) 3 species for the same purposes: Artemisia absinthium, Tanacetum vulgare and Tussilago farfara. The CAA, Código Alimentario Argentino ('Argentine Food Code') includes 9 species: Artemisia dracunculus, Cichorium intybus, Cnicus benedictus, Cynara cardunculus, Helianthus annuus, Matricaria chamomilla, Smallanthus sonchifolius, Stevia rebaudiana and Taraxacum officinale. This code prohibits the use of Artemisia absinthium. Also, ANMAT approves the use of Arctium lappa, Calendula officinalis and Smallanthus sonchifolius for dietary supplements produced in the country (ANMAT, 2013). Beyond these specifications, the species products mentioned can be purchased in the conurbation or through the Internet. 
Products circulation: The origin of the products is partially clear. Certain products carry a laboratory mark, but it is not explicit the raw materials source. In some cases, herbal products and its tinctures are presumably obtained from wild plants from native species or exotic naturalized (eg Achillea millefolium, Taraxacum officinale). Others products may come from plants grown in homegardens. According to informants interviewed, plants or parts thereof which are sold fresh in the Bolivian market, come from homegardens located in periurban areas of the conurbation (eg Baccharis species, Stevia rebaudiana). Bolivian immigrants working in horticultural practices in the periurban sector provide fresh plants to other immigrants of the same origin, which commercialized those plants in the market located in the urban sector. It is registered that Porophyllum ruderale and Tagetes minuta were cultivated in periurban homegardens from seeds brought from Bolivia (Pochettino et al. 2012a). Furthermore, in the Bolivian market of Liniers different products are entered directly from Bolivia, by land and quickly (48 to 72 hours), to the bus terminal of that neighborhood. Some fresh products could in this way get into the conurbation.

Products imported from Bolivia originally covered the demand for Bolivian and Peruvian communities in Buenos Aires, but in a short time some restricted products to immigrants (invisible) entered the general commercial circuit through the dietéticas. The most striking example is that of Smallanthus sonchifolius, which in less than a decade (Hurrell et al. 2013), and powered by the mass media, invaded the market with various products, specially dietary supplements. Under this type of product this species got his greatest visibility. Echinacea purpurea and Stevia rebaudiana are similar cases, but became visible in more than a decade. Other products derived from Asteraceae are visible from a long time in the metropolitan area, including the recent immigrants segments, eg Achyrocline satureioides, Arnica montana, Baccharis species and Matricaria chamomilla.

Uses assigned/Effects recorded: Table 1 presents a wide variety of uses assigned, some with roots in folk medicine, other promoted by the dietéticas and the media. It is noteworthy that the diffusion of the products often combines scientific information about the properties evaluated (generally, only some of them) with the nontraditional revaluation of the uses of plants coming from 'millenary traditions'. This combination of tradition and innovation apparently aims to capture the accessions of a diverse set of consumers, that is, a proper selling strategy for urban areas. An example is Smallanthus sonchifolius that stores fructooligosaccharides (polymers of fructose), carbohydrates with fewer calories than saccharose, so they do not increase the levels of glucose in the blood, resulting suitable for diabetics and lowcalorie diets. Scientific studies validate its traditional use (against diabetes) while making possible promoting a new urban use (for slimming).

Most scientific studies on the medicinal plants effects correspond to cases in vitro or in vivo in animals, so that clinical studies are required in humans. However, the assessment of potential effects is promise for future studies on the prevention and treatment of various diseases. For example, Haplopappus rigidus and Senecio eriophyton are commercialized to treat male sexual impotence. The activity of the extracts of these plants in animals was evaluated, and the results seem to validate their folk medicinal use, and like authors say: 'open new ways in the search for natural products with vasodilatory effects' (Hnatyszyn et al. 2003). It has to be highlighted that the results of many studies refers to the potential effects on humans, not the scientifically proven. In this context, many uses that are affirmed and diffused, specially through the Internet, contain inaccurate information. 
According to informants interviewed, the inquiries made by consumers about the products to be used refer to a wide range of possible effects. Many people flock to the dietéticas to acquire plant products to treat specific ailments: digestive, hepatic, diuretic, antidermatitis, antiarthritic, antirheumatic, and anti-inflammatory, among others. But also, many informants considered very relevant some plant products that are in high demand, probably due to the excessive media propaganda: slimming, aphrodisiacs and/or sexual performance optimizers, and against stress. These so widespread uses reveal an underlying set of requirements and interests related to living in large metropolitan areas.

Slimming products relate to the aesthetic aspects as well as therapeutic also, in order to treat obesity. Of the 50 Asteraceae species surveyed, $10(20 \%)$ are used for slimming: Achyrocline satureoides, Cichorium intybus, Cynara cardunculus, Helianthus annuus, Hieracium pilosella, Matricaria chamomilla, Schkuhria pinnata, Smallanthus sonchifolius, Stevia rebaudiana, and Taraxacum officinale. Of this group only one species, Cichorium intybus, has been identified for treatment against obesity (Vasudeva et al. 2012). In the other cases the use as slimming can be linked with other researched effects, eg diuretic, hypoglycemic, and hypolipidemic.

Human sexuality is a complex phenomenon that involves several aspects: biological, psychological, social, cultural. Also, the concept of aphrodisiac, as a stimulant of sexual desire, is associated with one or more of these aspects. In general, plant products considered aphrodisiacs are consumed to promote and facilitate sexual unions. Aphrodisiacs are also related with plant products considered sexual performance optimizers, in particular, those who act against male impotence. Of the 50 Asteraceae species surveyed, 8 of them (16\%) are used as aphrodisiacs: Artemisia absinthium, Baccharis articulata, B. crispa, B. trimera, Cynara cardunculus, Echinacea purpurea, Haplopappus rigidus, Tagetes minuta. Of these species, Baccharis articulata, B. crispa, B. trimera and Haplopappus rigidus are also used against male impotence. This use is likewise assigned to Senecio eriophyton. As mentioned before, only Haplopappus rigidus and Senecio eriophyton were surveyed about their use related to male impotence. Only one species, Gnaphalium gaudichaudianum, is considered anaphrodisiac.

It is noteworthy that sexuality also includes other aspects linked to human reproduction that involve other uses, such as contraceptive, abortifacient, against male and female infertility, against birth and postpartum ailments, postpartum recovery, neonatal care, galactogogue. Uses related to the reproductive organs should be also considered, as emmenagogue, antiamenorrheic, against specific diseases (eg prostate cancer) and venereal infections. This complex system of uses related to human reproduction will be grounds for a future contribution. However, it is remarkable the number of species used as abortifacient and/or contraceptive: Acanthospermum australe, Ambrosia elatior, Artemisia absinthium, Baccharis articulata, Baccharis trimera, Cnicus benedictus, Pluchea sagittalis, Stevia rebaudiana, Tanacetum vulgare, Trixis divaricata subsp. discolor, Tussilago farfara and Xanthium spinosum (12 species: $24 \%$ of the total). Only for Ambrosia elatior and Stevia rebaudiana its contraceptive activity was analyzed. One species, Tagetes minuta, is considered antiabortifacient.

Stress is the organism's response to situations perceived as threatening or as increased demands that may cause the appearance of functional anomalies and generate emotional dysfunctions, like mood alterations, nervousness, anxiety, insomnia, lost of memory and lack of concentration, among others. In this context, in treating against stress various uses such as sedative, anxiolytic, antidepressant, 
hypnotic, and/or mnemonic are included. These uses are assigned to 18 of the 50 Asteraceae species surveyed (36\%): Achyrocline satureoides, Helichrysum italicum, Mikania periplocifolia, Pluchea sagittalis, Senecio nutans, Tanacetum parthenium, Tussilago farfara, Xanthium spinosum (sedative), Acanthospermum australe, Acanthostyles buniifolius, Chrysanthemum morifolium, Senecio eriophyton (sedative, hypnotic), Achillea millefolium, Chamaemelum nobile (sedative, anxiolytic), Tagetes minuta (sedative, antidepressant), Tanacetum vulgare (sedative, mnemonic), Artemisia absinthium (hypnotic, mnemonic), and Matricaria chamomilla (sedative, anxiolytic, hypnotic, mnemonic). Of this set of species and uses assigned, the effects have been studied for only 6 species: Matricaria chamomilla (sedative, anxiolytic, hypnotic, mnemonic), Chamaemelum nobile (sedative, anxiolytic), Acanthostyles buniifolius and Chrysanthemum morifolium (hypnotic), Achillea millefolium (anxiolytic) and Tagetes minuta (antidepressant). Matricaria chamomilla is the species most widespread and more frequently commercialized for these purposes.

Finally, although it is true that for many of the species assessed there are information about its effects and biological activity (eg Achyrocline satureoides, Matricaria chamomilla, Smallanthus sonchifolius, Stevia rebaudiana), for other species would be required different studies to validate the uses assigned to them (eg Baccharis and Senecio species, Tessaria integrifolia, Trixis divaricata subsp. discolor). In this sense, it is known that the basic ethnobotanical researches make it possible a register of species and its therapeutic uses, to guide the search of new active principles with pharmacological applications. This is valid for both traditional uses and the uses assigned in urban scenarios. Facing the future, some significant effects scientifically recorded for Asteraceae are: antimicrobial (43 species: $86 \%$ of the total), antioxidant (38 species: $76 \%$ ), antitumor and cytotoxic (35 species: $70 \%$ ), antiviral (20 species: $40 \%$, including anti-HIV: eg Acanthostyles buniifolius, Calendula officinalis, Chrysanthemum morifolium), hypoglycemic and antidiabetic (15 species: $30 \%$ ), immunomodulatory and immunostimulant (13 species: $26 \%$ ), hipolipidemic and hypocholesterolemic (6 species: 12\%).

\section{Conclusions}

This contribution shows results of ongoing research line on urban ethnobotany related to medicinal and/or aromatic Asteraceae in the conurbation Buenos Aires-La Plata, Argentina. Table 1 itself is a description of the available data on species, its products, uses assigned and effects and biological activity studied. About the species and its products, the data come from fieldwork. Assigned uses are derived from both fieldworks as the literature reviewed and the information presented in different labels, leaflets, catalogs and advertisements. The biological activity and the effects studied come from the literature review.

Data interpretation aims to assess the composition of urban botanical knowledge in relation to plant products circulating. For this, it is assumed that the botanical knowledge guides the selection of those products. The species and products exclusive of the immigrant segments (Bolivian and Chinese) are linked to their traditions of origin, although the same species may be represented in nontraditional commercial circuit through other products. For evaluated Asteraceae, fresh plants or parts thereof of Arctium lappa, Baccharis articulata or Matricaria chamomilla are examples of exclusive products that are related to knowledge linked to traditions (invisible for most of urban population). But herbal products or tinctures derived of those species are frequent plants products into the dietéticas, hence are 
visible for most of urban population. Smallanthus sonchifolius and Stevia rebaudiana are examples of species whose very varied products are sold in the general commercial circuit, ie are visible. However the presence of fresh products in the Bolivian market (invisible) suggests that species have gained visibility in the urban scenario. From a temporal standpoint, the visualization process is fast in pluricultural contexts: just in one or two decades the products of those species have invaded the general commercial circuit. Its high demand is due to the mass media, including the Internet, that disseminate its uses. On the other hand, some species remain until now invisible, eg Porophyllum ruderale, only found in the Bolivian market, despite having active compounds with diverse interesting effects, like antimicrobial, antinociceptive, anti-inflammatory, cytotoxic.

The role of the media is expansive on the botanical knowledge and guides the selection of plant products. But, at the same time, offer new sale arguments (as new uses derived from scientific studies or from traditional uses little known before) and acquire a recursive role: the knowledge guides the consumption which increases the demand, and this feeds back on knowledge, which reorients the consumption. For example, Smallanthus sonchifolius was traditionally used for cases of diabetes, and people know now that it also has hypoglycemic effect and may be considered as slimming, as well as a promising phytotherapic medicine for antitumor treatments. This recursiveness between knowledge and behavior is at the base of the urban botanical knowledge dynamics, in which ancient uses are resignified and new uses are added into the urban context. Thereby, the botanical knowledge system adapts and evolves.

The Asteraceae family, with so diverse applications, particularly as aromatic and/or medicinal plants, is an interesting reference group to obtain information about the composition and dynamic of urban botanical knowledge. As well as it has the major number of native taxa used in popular medicine in Argentina, also it has the highest number of taxa whose products are marketed in the metropolitan Buenos Aires-La Plata. In connection with the uses assigned and the effects analyzed, although many species have been the subject of several evaluations about its principles with biological activity, even many other species need to be evaluated. Data provided by this contribution may encourage further studies in this direction. Thus it may provide a more solid basis for many uses assigned and above all, avoid misinformation about the medicinal properties of many species.

\section{Acknowledgments}

The authors wish to thank to Alejandro $\mathrm{C}$. Pizzoni for his critical reading of the manuscript, to Professor Emilio Ulibarri for his help in fieldworks, and to Dr. María Lelia Pochettino for her continued support, and through her to all collaborators of the LEBA. Ongoing research was partially carried out with financial support of the Consejo Nacional de Investigaciones Cientificas y Técnicas (CONICET) of Argentina.

\section{References}

AABA (2013) Atlas Ambiental de Buenos Aires. Museo Argentino de Ciencias Naturales [http://www.atlasdebuenosaires.gov.ar] Accessed 25 April 2013.

Abdelhamed S, Yokoyama S, Hafiyani L, Kalauni SK, Hayakawa Y, Awale S, Saiki I (2013) Identification of plant extracts sensitizing breast cancer cells to TRAIL. Oncology Reports 2013, doi: 10.3892/or.2013.2293. 
Adams M, Gmunder F, Hamburger M (2007) Plants traditionally used in age related brain disorders. A survey of ethnobotanical literature. Journal of Ethnopharmacology 113(3):363-381.

Aguilar F, Bonilla P (2009) Actividad antioxidante e inmunológica de flavonoides aislados de hojas de Smallanthus sonchifolius (yacón). Ciencia e Investigación 12(1):15-23.

Akihisa T, Yasukawa K, Oinuma H, Kasahara Y, Yamanouchi S, Takido M, Kumaki K, Tamura T (1996) Triterpene alcohols from the flowers of compositae and their anti-inflammatory effects. Phytochemistry 43(6):1255-1260.

Akihisa T, Franzblau SG, Ukiya M, Okuda H, Zhang F, Yasukawa K, Suzuki T, Kimura Y (2005) Antitubercular activity of triterpenoids from Asteraceae flowers. Biological and Pharmaceutical Bulletin 28(1):158-160.

Al-Musayeib NM, Mothana RA, Matheeussen A, Cos P, Maes L (2012) In vitro antiplasmodial, antileishmanial and antitrypanosomal activities of selected medicinal plants used in the traditional Arabian Peninsular region. BMC Complementary and Alternative Medicine 12:49, doi: 10.1186/1472-6882-12-49.

Albuquerque U, Lucena RF (2004) Métodos e técnicas na pesquisa etnobotânica. NUPEEA, Recife.

Albuquerque UP, Hurrell JA (2010) Ethnobotany: one concept and many interpretations. In: Albuquerque UP, Hanazaki N (eds) Recent developments and case studies in Ethnobotany. SBEE/NUPEEA, Recife, pp. 87-99.

Alexíades MN, Sheldon JW (1996) Selected guidelines for ethnobotanical research: a field manual. The New York Botanical Garden, New York.

Almeida AB, Sánchez-Hidalgo M, Martín AR, Luiz-Ferreira A, Trigo JR, Vilegas W, dos Santos LC, Souza-Brito AR, de la Lastra CA (2013) Anti-inflammatory intestinal activity of Arctium lappa L. (Asteraceae) in TNBS colitis model. Journal of Ethnopharmacology 146(1):300-310.

Alonso J (2005) Monografía: Baylahuén, Haplopappus rigidus Phil. Boletín Latinoamericano y del Caribe de Plantas Medicinales y Aromáticas 4(3):60-63.

Alonso J. Desmarchelier C (2005) Plantas medicinales autóctonas de la Argentina. Lola, Buenos Aires.

Álvarez A, Habtemariam S, Juan-Badaturuge M, Jackson C, Parra F (2011) In vitro anti HSV-1 and HSV-2 activity of Tanacetum vulgare extracts and isolated compounds: An approach to their mechanisms of action. Phytotherapy Research 25(2):296-301.

Alves D (1996) Estudo fitoquímico e efeitos analgésicos da Porophyllum ruderale. Universidade Estadual de Campinas, Piracicaba.

Amat N, Upur H, Blazeković B (2010) In vivo hepatoprotective activity of the aqueous extract of Artemisia absinthium $\mathrm{L}$. against chemically and immunologically induced liver injuries in mice. Journal of Ethnopharmacology 131(2):478-484.

Amsterdam JD, Shults J, Soeller I, Mao JJ, Rockwell K, Newberg AB (2012) Chamomile (Matricaria recutita) may provide antidepressant activity in anxious, depressed humans: an exploratory study. Alternative Therapies in Health and Medicine 18(5):44-49.

Aqil F, Ahmad I (2007) Antibacterial properties of traditionally used Indian medicinal plants. Methods and Findings in Experimental and Clinical Pharmacology 29(2):79-92. 
Arancibia L, Naspi C. Pucci G, Arce M (2013) Biological activity of a furanoeremophilane isolated from Senecio filaginoides var. filaginoides. Boletín Latinoamericano y del Caribe de Plantas Medicinales y Aromáticas 12(1):18-23.

Arenas PM, Cristina I, Puentes JP, Buet Costantino F, Hurrell JA, Pochettino ML (2011) Adaptógenos: plantas medicinales tradicionales comercializadas como suplementos dietéticos en la conurbación Buenos Aires-La Plata (Argentina). Bonplandia (Corrientes) 20(2):251-264.

Atta A, Elkoly T, Mouneir S, Kamel G, Alwabel N, Zaher S (2010) Hepatoprotective effect of methanol extracts of Zingiber officinale and Cichorium intybus. Indian Journal of Pharmaceutical Sciences 72(5):564-570.

Ayvar M, Sánchez Riera A, Grau A, Sánchez S (2001) Hypoglycemic effect of the water extract of Smallanthus sonchifolius (yacon) leaves in normal and diabetic rats. Journal of Ethnopharmacology 74(2):125-132.

Bader G, Plohmann B, Hiller K, Franz G (1996) Cytotoxicity of triterpenoid saponins. 1: Activities against tumor cells in vitro and hemolytical index. Pharmazie 5(6):414-417.

Bail S, Buchbauer G, Jirovetz L, Denkova Z, Slavchev A, Stoyanova A, Schmidt E, Geissler M (2009) Antimicrobial activities of Roman Chamomile oil from France and its main compounds. Journal of Essential Oil Research 21(3):283-286.

Barboza G, Cantero J, Nuñez CO, Pacciaroni A, Ariza Espinar L (2009) Medicinal plants: A general review and a phytochemical and ethnopharmacological screening of the native Argentine Flora. Kurtziana 34(1-2):7-365.

Bardón A, Borkosky S, Ybarra MI, Montanaro S, Cartagena E (2007) Bioactive plants from Argentina and Bolivia. Fitoterapia 78(3):227-231.

Baretta I, Felizardo R, Bimbato V, dos Santos M, Kassuya C, Gasparotto J, da Silva C, de Oliveira S, Ferreira J, Andreatini R (2012) Anxiolytic-like effects of acute and chronic treatment with Achillea millefolium L. extract. Journal of Ethnopharmacology 140(1):46-54.

Barioni ED, Santin JR, Machado ID, Rodrigues SF, Ferraz-de-Paula V, Wagner TM, Cogliati B, Corrêa Dos Santos M, Machado Mda S, de Andrade SF, Niero R, Farsky SH (2013) Achyrocline satureioides (Lam.) D.C. Hydroalcoholic extract inhibits neutrophil functions related to innate host defense. EvidBased Complementary and Alternative Medicine 2013, doi: 10.1155/2013/787916.

Barsky A (2005) El periurbano productivo: un espacio en constante transformación. Introducción al estado del debate y referencias al caso de Buenos Aires. Scripta Nova (Barcelona) 9(194):36 (1 August 2005).

Barsky A (2010) La agricultura de 'cercanías' a la ciudad y los ciclos del territorio periurbano. Reflexiones sobre el caso de la Región Metropolitana de Buenos Aires. In Svetlitza de Nemirovsky (ed), Agricultura periurbana en Argentina y globalización. FLACSO, Buenos Aires, pp. 15-29.

Bauer J, Dehm F, Koeberle A, Pollastro F, Appendino G, Rossi A, Sautebin L, Werz $O$ (2010) In vitro and in vivo evaluation of the anti-inflammatory effects of arzanol from Helichrysum italicum. Planta Medica 76:P071, doi: 10.1055/s0030-1264369.

Beaux D, Fleurentin J, Mortier F (1999) Effect of extracts of Orthosiphon stamineus, Hieracium pilosella, Sambucus nigra and Arctostaphylos uva-ursi in rats. Phytotherapy Research 13(3):222-225. 
Benedek B, Geisz N, Jäger W, Thalhammer T, Kopp B (2006) Choleretic effects of yarrow (Achillea millefolium s.I.) in the isolated perfused rat liver. Phytomedicine 13(9-10):702-706.

Benli M, Kaya I, Yigit N (2007) Screening antimicrobial activity of various extracts of Artemisia dracunculus L. Cell Biochemistry and Function 25(6):681-686.

Bhakuni DS, Bittner M, Marticorena C, Silva M, Weldt E, Hoeneisen M (1976) Screening of Chilean plants for anticancer activity. Lloydia 39:225-243.

Bogado Bordazar L (2003) Migraciones internacionales: influencia de la migración china en Argentina y Uruguay. Thesis 12. Facultad de Ciencias Jurídicas y Sociales, Universidad Nacional de La Plata, La Plata.

Bongiovanni G, Luchino N, Palacio L, Eynard A, Cantero J, Goleniowski M (2006) In vitro antitumoral activity determination of native plant extracts of the central region of Argentina. Molecular Medicinal Chemistry 10:22-23.

Boonkaewwan C, Toskulkao C, Vongsakul M (2006) Anti-Inflammatory and immunomodulatory activities of stevioside and its metabolite steviol on THP-1 cells. Journal of Agricultural and Food Chemistry 54(3):785-789.

Bora KS, Sharma A (2010) Neuroprotective effect of Artemisia absinthium L. on focal ischemia and reperfusion-induced cerebral injury. Journal of Ethnopharmacology 129(3):403-409.

Broide DH (2009) Immunomodulation of allergic disease. Annual Review of Medicine 60:279-291.

Bucciarelli A, Skliar Ml (2007) Plantas medicinales de Argentina con actividad gastroprotectora. Ars Pharmaceutica 48(4):361-369.

Bukhari IA, Shah AJ, Khan RA, Meo SA, Khan A, Gilani AH (2013) Gut modulator effects of Conyza bonariensis explain its traditional use in constipation and diarrhea. European Review for Medical and Pharmacological Sciences 17(4):552-558.

Burgstaller $\mathrm{CH}$ (1968) La vuelta a los vegetales. 2nd ed. Burgstaller, Buenos Aires.

Bussmann RW, Sharon D, Díaz D, Barocio Y (2008) Peruvian plants canchalagua (Schkuhria pinnata (Lam.) Kuntze), hercampuri (Gentianella alborosea (Gilg.) Fabris), and corpus way (Gentianella bicolor (Wedd.) J. Pringle) prove to be effective in the treatment of acne. Arnaldoa 15(1):149-152.

Butnariu M, Coradini C (2012) Evaluation of biologically active compounds from Calendula officinalis flowers using spectro-photometry. Chemistry Central Journal 6(1):35.

Canlas J, Hudson JB, Sharma M, Nandan D (2010) Echinacea and trypanasomatid parasite interactions: Growth-inhibitory and anti-inflammatory effects of Echinacea. Pharmaceutical Biology 48(9):1047-1052.

Carvalho LH, Krettli AU (1991) Antimalarial chemotherapy with natural products and chemically defined molecules. Memórias do Instituto Oswaldo Cruz 86(Suppl 2):181-184.

Casero C, Estévez-Braun A, Ravelo AG, Demo M, Méndez-Álvarez S, Machín F (2013) Achyrofuran is an antibacterial agent capable of killing methicillinresistant vancomycin-intermediate Staphylococcus aureus in the nanomolar range. Phytomedicine 20(2):133-138.

Cemek M, Kağa S, Şimşek N, Büyüko M, Konuk M (2008) Antihyperglycemic and antioxidative potential of Matricaria chamomilla in streptozotocininduced diabetic rats. Journal of Natural Medicines 62(3):284-293. 
Cerrutti M (2009) Diagnóstico de las poblaciones de inmigrantes en la Argentina. Dirección Nacional de Población, Buenos Aires.

Ceuterick M, Vandebroek I, Torrya B, Pieroni, A (2008) Cross-cultural adaptation in urban ethnobotany. The Colombian folk pharmacopoeia in London. Journal of Ethnopharmacology 120(3): 342-359.

Chalchat JC, Maksimović Z, Petrović S, Gorunović M, Đorđević S, Mraović M (2004) Chemical composition and antimicrobial activity of Ambrosia artemisiifolia L. essential oil. Journal of Essential Oil Research 16:270-273.

Chan YS, Cheng LN, Wu JH, Chan E, Kwan YW, Lee SM, Leung GP, Yu PH, Chan SW (2011) A review of the pharmacological effects of Arctium lappa. Inflammopharmacology 19(5):245-254.

Chandrashekhar VM, Halagali KS, Nidavani RB, Shalavadi MH, Biradar BS, Biswas D, Muchchandi IS (2011) Anti-allergic activity of German chamomile (Matricaria recutita L.) in mast cell mediated allergy model. Journal of Ethnopharmacology 137(1):336-340.

Chen T, Chen S, Chan P, Chu Y, Yang H, Cheng J (2005) Mechanism of the hypoglycemic effect of stevioside, a glycoside of Stevia rebaudiana. Planta Medica 7:108-113.

Cheung C, Gibbons N, Johnson D, Nicol D (2010) Silibinin, a promising new treatment for cancer. Anti-Cancer Agents in Medicinal Chemistry 10(3):186195.

Cho J, Kim H, Ryu J, Jeong Y, Lee Y, Jin C (2005) Neuroprotective and antioxidant effects of the ethyl acetate fraction prepared from Tussilago farfara L. Biological and Pharmaceutical Bulletin 28(3):455-460.

Choi JG, Kang OH, Lee YS, Oh YC, Chae HS, Obiang-Obounou B, Park SC, Shin D, Hwang BY, Kwon DY (2010) Antimicrobial activity of the constituents of Smallanthus sonchifolius leaves against methicillin-resistant Staphylococcus aureus. European Review for Medical and Pharmacological Sciences 14(12):1005-1009.

Choi UK, Lee OH, Yim JH, Cho CW, Rhee YK, Lim SI, Kim YC (2010) Hypolipidemic and antioxidant effects of dandelion (Taraxacum officinale) root and leaf on cholesterol-fed rabbits. International Journal of Molecular Sciences 11(1):67-78.

Chun H, Kitts D (2003) Antioxidant, pro-oxidant, and cytotoxic activities of solvent-fractionated dandelion flower extracts in vitro. Journal of Agricultural and Food Chemistry 51:301-310.

Conforti F, loele G, Statti G, Marrelli M, Ragno G, Menichin F (2008) Antiproliferative activity against human tumor cell lines and toxicity test on Mediterranean dietary plants. Food and Chemical Toxicology 46(10):33253332.

Cosentino M, Bombelli R, Carcano E, Luini A, Marino F, Crema F, Dajas F, Lecchini S (2008) Immunomodulatory properties of Achyrocline satureioides (Lam.) DC. infusion: a study on human leukocytes. Journal of Ethnopharmacology 116(3):501-507.

Craciunescu O, Constantin D, Gaspar A, Toma L, Utoiu E, Moldovan L (2012) Evaluation of antioxidant and cytoprotective activities of Arnica montana L. and Artemisia absinthium L. ethanolic extracts. Chemistry Central Journal 6(1):97.

Csupor-Löffler B, Hajdú Z, Zupkó I, Réthy B, Falkay G, Forgo P, Hohmann J (2009) Antiproliferative effect of flavonoids and sesquiterpenoids from Achillea 
millefolium on cultured human tumour cell lines. Phytotherapy Research 23(5):672-676.

Cunningham AB (2001) Etnobotánica Aplicada. Pueblos, uso de plantas silvestres y conservación. Nordan-Comunidad, Montevideo.

Dadé MM, Fioravanti DE, Schinella GR, Tournier HA (2009) Total antioxidant capacity and polyphenol content of 21 aqueous extracts obtained from native plants of Traslasierra valley (Argentina). Boletín Latinoamericano y del Caribe de Plantas Medicinales y Aromáticas 8(6):529-539.

Dall'Acqua S, Bolego C, Cignarella A, Gaion RM, Innocenti G (2011) Vasoprotective activity of standardized Achillea millefolium extract. Phytomedicine 18(12):1031-1036.

Das SK, Mukherjee S (2012) Biochemical and immunological basis of silymarin effect, a milk thistle (Silybum marianum) against ethanol-induced oxidative damage. Toxicology Mechanisms and Methods 22(5):409-413.

Davicino R, Mattar M, Casali Y, Correa S, Pettenati E, Micalizzi B (2007) Actividad antifúngica de extractos de plantas usadas en medicina popular en Argentina. Revista Peruana de Biología 14(2):247-251.

Delgado GT, Thomé R, Gabriel DL, Tamashiro WM, Pastore GM (2012) Yacon (Smallanthus sonchifolius) derived fructooligosaccharides improves the immune parameters in the mouse. Nutrition Research 32(11):884-892.

Desmarchelier C, Novoa Bermúdez MJ, Coussio JD, Nadinic EL, Debenedetti SL, Ciccia C, Boveris A (1994) Búsqueda de nuevos antioxidantes naturales. Anales Sociedad Argentina para la Investigación de Productos Aromáticos 14:315-319.

Deutschländer M, van de Venter M, Roux S, Louw J, Lal N (2009) Hypoglycemic activity of four plant extracts traditionally used in South Africa for diabetes. Journal of Ethnopharmacology 124(3):619-624.

Di Pierro F, Callegari A, Carotenuto D, Mollo Tapia M (2008) Clinical efficacy, safety and tolerability of BIO-C (micronized silymarin) as a galactagogue. Acta Biomedica 79:205-210.

Dos Santos AC, Baggio CH, Freitas CS, Lepieszynski J, Mayer B, Twardowschy A, Missau FC, Santos EP, Pizzolatti MG, Marques MC (2008) Gastroprotective activity of the chloroform extract of the roots from Arctium lappa $L$. Journal of Pharmacy and Pharmacology 60(6):795-801.

Duarte CM, Quirino MR, Patrocínio MC, Anbinder AL (2011) Effects of Chamomilla recutita (L.) on oral wound healing in rats. Medicina Oral, Patologia Oral y Cirugia Bucal 16(6):e716-e721.

Duarte MC, Figueira GM, Sartoratto A, Rehder VL, Delarmelina C (2005) AntiCandida activity of Brazilian medicinal plants. Journal of Ethnopharmacology 97(2):305-311.

El Zalabani SM, Hetta MH, Ismail AS (2012) Genetic profiling, chemical characterization and biological evaluation of two Conyza species growing in Egypt. Journal of Applied Pharmaceutical Science 2(11):054-061.

Emendörfer F, Emendörfer F, Bellato F, Noldin VF, Cechinel-Filho V, Yunes RA, Delle Monache F, Cardozo AM (2005) Antispasmodic activity of fractions and cynaropicrin from Cynara scolymus on guinea-pig ileum. Biological and Pharmaceutical Bulletin 28(5):902-904.

Espiña DC, Carvalho FB, Zanini D, Schlemmer JB, Coracini JD, Rubin MA, Morsch VM, Schetinger MR, Leal DB, Baiotto CR, Jaques JA (2012) A more accurate 
profile of Achyrocline satureioides hypocholesterolemic activity. Cell Biochemistry and Function 30(4): 347-353.

Falconieri D, Piras A, Porcedda S, Marongiu B, Gonçalves M, Cabral C, Cavaleiro C, Salgueiro $L$ (2011) Chemical composition and biological activity of the volatile extracts of Achillea millefolium. Natural Product Communications 6(10):1527-1430.

Falleh H, Ksouri R, Chaieb K, Karray-Bouraoui N, Trabelsi N, Boulaaba M, Abdelly C (2008) Phenolic composition of Cynara cardunculus L. organs, and their biological activities. Comptes Rendus Biologies 331(5):372-379.

Fantini N, Colombo G, Giori A, Riva A, Morazzoni P, Bombardelli E, Carai MA (2011) Evidence of glycemia-lowering effect by a Cynara scolymus L. extract in normal and obese rats. Phytotherapy Research 25(3):463-466.

Fernández T, Cerdá Zolezzi P, Risco E, Martino V, López P, Clavin M, Hnatyszyn O, Canigueral S, Hajos S, Ferraro G, Alvarez E (2002) Immunomodulating properties of Argentine plants with ethnomedicinal use. Phytomedicine $9(6): 546-552$.

Figueredo SM, do Nascimento FP, Freitas CS, Baggio CH, Soldi C, Pizzolatti MG, de Ibarrola Mdel C, Arrua RL, Santos AR (2011) Antinociceptive and gastroprotective actions of ethanolic extract from Pluchea sagittalis (Lam.) Cabrera. Journal of Ethnopharmacology 135(3):603-609.

Fischedick JT, Standiford M, Johnson DA, De Vos RC, Todorović S, Banjanac T, Verpoorte R, Johnson JA (2012) Activation of antioxidant response element in mouse primary cortical cultures with sesquiterpene lactones isolated from Tanacetum parthenium. Planta Medica 78(16):1725-1730.

Fonseca Y, Catini C, Vicentini F, Nomizo A, Gerlach R, Fonseca M (2010) Protective effect of Calendula officinalis extract against UVB-induced oxidative stress in skin: evaluation of reduced glutathione levels and matrix metalloproteinase secretion. Journal of Ethnopharmacology 127(3):596-601.

Forstall RL, Greene RP, Pick JB (2004) Which are the largest? City Futures Conference, University of Illinois, Chicago.

Foster JG, Cassida K, Turner K (2011) In vitro analysis of the anthelmintic activity of forage chicory (Cichorium intybus) sesquiterpene lactones against a predominantly Haemonchus contortus egg population. Veterinary Parasitology 180:298-306.

Freire SE, Urtubey E (1999a). Compuestas Medicinales de la Provincia Biogeográfica Pampeana. Claves para su Determinación e Iconografías. Parte I. Acta Farmacéutica Bonaerense 18(3):191-199.

Freire SE, Urtubey E (1999b). Compuestas Medicinales de la Provincia Biogeográfica Pampeana. Claves para su Determinación e Iconografías. Parte II. Acta Farmacéutica Bonaerense 18(4):283-294.

Freire SE, Urtubey E (2000a) Compuestas Medicinales de la Provincia Biogeográfica Pampeana. Claves para su Determinación e Iconografías. Parte III. Acta Farmacéutica Bonaerense 19(1):13-16.

Freire SE, Urtubey E (2000b) Compuestas Medicinales de la Provincia Biogeográfica Pampeana. Claves para su Determinación e Iconografías. Parte IV. Acta Farmacéutica Bonaerense 19(2):85-90.

Freire SE, Urtubey E (2000c) Compuestas Medicinales de la Provincia Biogeográfica Pampeana. Claves para su Determinación e Iconografías. Parte V. Acta Farmacéutica Bonaerense 19(3):165-184. 
Frey FM, Meyers R (2010) Antibacterial activity of traditional medicinal plants used by Haudenosaunee peoples of New York State. BMC Complementary and Alternative Medicine 10:64, doi: 10.1186/1472-6882-10-64.

Gawronska-Grzywacz M, Krzaczek T (2006) Sterol composition from inflorescens of Hieracium pilosella L. Acta Societatis Botanicorum Poloniae 75(1):29-32.

Gawronska-Grzywacz M, Krzaczek T, Nowak R, Los, R, Malm A, Cyranka M, Rzeski W (2011) Biological activity of new flavonoid from Hieracium pilosella L. Central European Journal of Biology 6(3):27-404.

Gemini M (2003) ¿Qué es el Gran Buenos Aires? Instituto Nacional de Estadística y Censos (INDEC), Buenos Aires.

Gené RM, Marin E, Adzet T (1992) Anti-inflammatory effect of aqueous extracts of three species of the genus Baccharis. Planta Medica 58:565-566.

Gené RM, Cartaña C, Adzet T, Marin E, Parella T, Cañigueral S (1996) AntiInflammatory and analgesic activity of Baccharis trimera: Identification of its active constituents. Planta Medica 62:232-235.

Ghaemi A, Soleimanjahi H, Farsbaf Mogahddam M, Omidbaigi R, Pourbaig MVM (2004) Antiviral activity of root extracts from Tagetes minuta against Herpes simplex virus (HSV-1). Iranian Journal of Pharmaceutical Research 3(Suppl. 2):72.

Ghonime M, Eldomany R, Abdelaziz A, Soliman $H$ (2011) Evaluation of immunomodulatory effect of three herbal plants growing in Egypt. Immunopharmacology and Immunotoxicology 33(1):141-145.

Giada MD, Mancini-Filho J (2009) Antioxidant capacity of the striped sunflower (Helianthus annuus) seed extracts evaluated by three in vitro methods. International Journal of Food Sciences and Nutrition 60(5):395-401.

Gianello JC, Cenal JP, Giordano OS, Tonn CE, Petenatti ME, Petenatti EM, Del Vitto LA (2000). Medicamentos herbarios en el Centro-oeste Argentino. II. 'Carquejas": control de calidad de las drogas oficiales y sustituyentes. Acta Farmacéutica Bonaerense 19(2):99-103.

Gil JC, Lingan P, Flores C, Chimoy PJ (2008) Efecto a largo plazo del consumo de Stevia rebaudiana (Magnoliopsida, Asteraceae) en la fertilidad de ratones. Revista Peruana de Biología 15(1):85-90.

Ginesta-Peris E, Garcia-Breijo FJ, Primo-Yúfera E (1994) Antimicrobial activity of xanthatin from Xanthium spinosum L. Letters in Applied Microbiology 18:206-208.

Gonzales Dávalos E, Villca Jiménez T, Loza Almanza R (2007) Evaluación de la actividad antiinflamatoria de ocho especies del género Baccharis: $B$. articulata, B. dracunculifolia, B. salicifolia, B. ulicina, B. latifolia, B. pentlandii, B. obtusifolia, B. subulata. Revista Boliviana de Química 24(1):41-44.

Guglielmone HA, Nuñez-Montoya SC, Agnese AM, Pellizas CG, Cabrera JL, Donadio AC (2012) Quercetin 3,7,3',4'-tetrasulphated isolated from Flaveria bidentis inhibits tissue factor expression in human monocyte. Phytomedicine 19(12):1068-1071.

Gugliucci A, Menini T (2002) Three different pathways for human LDL oxidation are inhibited in vitro by water extracts of the medicinal herb Achyrocline satureoides. Life Sciences 71(6):693-705.

Guimarães R, Barros L, Dueñas M, Calhelha RC, Carvalho AM, Santos-Buelga C, Queiroz MJ, Ferreira IC (2013) Nutrients, phytochemicals and bioactivity 
of wild Roman chamomile: A comparison between the herb and its preparations. Food Chemistry 136(2):718-725.

Gutiérrez Durán MP, Limachi Viadez G, Gonzales Dávalos E, Bermejo Benito P (2011) Control de Calidad del Xanthium spinosum, planta medicinal expendida en la ciudad de La Paz, Bolivia. BIOFARBO 19(1):15-21.

Habib NC, Honoré SM, Genta SB, Sánchez SS (2011) Hypolipidemic effect of Smallanthus sonchifolius roots on diabetic rats: Biochemical approach. Chemico-Biological Interactions 194(1):31-39.

Hayashi K, Narutaki K, Nagaoka Y, Hayashi T, Uesato S (2010) Therapeutic effect of arctiin and arctigenin in immunocompetent and immunocompromised mice infected with influenza A virus. Biological and Pharmaceutical Bulletin 33(7):1199-1205.

Heng S, Harris KM, Kantrowitz ER (2010) Designing inhibitors against fructose 1,6-bisphosphatase: Exploring natural products for novel inhibitor scaffolds. European Journal of Medicinal Chemistry 45(4):1478-1484.

Heo JC, Woo SU, Kweon MA, Park JY, Lee HK, Son M, Rho JR, Lee SH (2008) Aqueous extract of the Helianthus annuus seed alleviates asthmatic symptoms in vivo. International Journal of Molecular Medicine 21(1):57-61.

Heywood VH, Harborne JB, Turner BL (1977) The Biology and Chemistry of the Compositae I. Academic Press, London-New York.

Hieronymus, J (1882) Plantae Diaphoricae Florae Argentinae. Kraft, Buenos Aires. Hnatyszyn O, Broussalis A, Herrera G, Muschietti L, Coussio J, Martino V, Ferraro G, Font M, Monge A, Martínez-Irujo JJ, Sanromán M, Cuevas MT, Santiago E, Lasarte JJ (1999) Argentine plant extracts active against polymerase and ribonuclease $\mathbf{H}$ activities of $\mathrm{HIV}-1$ reverse transcriptase. Phytotherapy Research 13(3):206-209.

Hnatyszyn O, Moscatelli V, Garcia J, Rondina R, Costa M, Arranz C, Balaszczuk A, Ferraro G, Coussio JD (2003) Argentinian plant extracts with relaxant effect on the smooth muscle of the corpus cavernosum of guinea pig. Phytomedicine 10(8):669-674.

Hnatyszyn O, Moscatelli V, Rondina R, Costa M, Arranz C, Balaszczuk A, Coussio J, Ferraro G (2004) Flavonoids from Achyrocline satureioides with relaxant effects on the smooth muscle of Guinea pig corpus cavernosum. Phytomedicine 11:366-369.

Honoré SM, Cabrera WM, Genta SB, Sánchez SS (2012) Protective effect of yacon leaves decoction against early nephropathy in experimental diabetic rats. Food and Chemical Toxicology 50(5):1704-1715.

Howes MR, Perry NS, Houghton PJ (2003) Plants with traditional uses and activities, relevant to the management of Alzheimer's disease and other cognitive disorders. Phytotherapy Research 17:1-18.

Hudson JB (2012) Applications of the phytomedicine Echinacea purpurea in infectious diseases. Journal of Biomedicine and Biotechnology 2012, doi: $10.1155 / 2012 / 769896$.

Hurrell JA, Albuquerque UP (2012). Is Ethnobotany an Ecological Science? Steps towards a complex Ethnobotany. Ethnobiology and Conservation 1:4 (4 October 2012).

Hurrell JA, Ulibarri, EA, Puentes JP, Buet-Costantino F, Arenas PM, Pochettino ML (2011a) Leguminosas medicinales y alimenticias utilizadas en la conurbación Buenos Aires-La Plata, Argentina. Boletín Latinoamericano y del Caribe de Plantas Medicinales y Aromáticas 10(5):443-455. 
Hurrell JA, Buet Costantino F, Puentes JP, Ulibarri EA, Pochettino ML (2011b). Huertos familiares periurbanos de las costas de Ensenada-Berisso y de la Isla Martín García (Buenos Aires, Argentina). Bonplandia (Corrientes) 20(2):213-229.

Hurrell JA, Pochettino ML, Puentes JP, Arenas PM (2013) Del marco tradicional al escenario urbano: Plantas ancestrales devenidas suplementos dietéticos en la conurbación Buenos Aires-La Plata, Argentina. Boletín Latinoamericano y del Caribe de Plantas Medicinales y Aromáticas (in press).

Hwangbo C, Lee H, Park J, Choe J, Lee J (2009) The anti-inflammatory effect of tussilagone, from Tussilago farfara, is mediated by the induction of heme oxygenase-1 in murine macrophages. International Immunopharmacology 9(13-14):1578-1584.

Ickes GR, Fong HH, Schiff PL, Perdue RE, Farnsworth NR (1973) Antitumor activity and preliminary phytochemical examination of Tagetes minuta (Compositae). Journal of Pharmaceutical Sciences 62(6):1009-1011.

INDEC. 2013. Instituto Nacional de Estadística y Censos. República Argentina. [http://www.indec.gov.ar/] Accessed 25 April 2013.

Irshad S, Mannan A, Mirza B (2011) Antimalarial activity of three Pakistani medicinal plants. Pakistan Journal of Pharmaceutical Sciences 24(4):589591.

Jamalian A, Shams-Ghahfarokhi M, Jaimand K, Pashootan N, Amani A, RazzaghiAbyaneh M (2012) Chemical composition and antifungal activity of Matricaria recutita flower essential oil against medically important dermatophytes and soil-borne pathogens. Journal de Mycologie Médicale 22(4):308-315.

Jawla S, Mogla2 OP, Kumar Y (2010) Herbal remedies for asthma: An overview. Journal of Chemical and Pharmaceutical Research 2(1): 267-272.

Jeon $\mathrm{H}$, Kang $\mathrm{H}$, Jung $\mathrm{H}$, Kang Y, Lim C, Kim Y, Park E (2008) Anti-inflammatory activity of Taraxacum officinale. Journal of Ethnopharmacology 115:82-88.

Juan-Badaturuge M, Habtemariam S, Jackson C, Thomas M (2009) Antioxidant principles of Tanacetum vulgare L. aerial parts. Natural Product Communications 4(11):1561-1564.

Kadarian C, Broussalis A, Miño J, López P, Gorzalczany S, Ferraro G, Acevedo C (2002) Hepatoprotective activity of Achyrocline satureioides. Pharmacological Research 45:57-61.

Kalvatchev Z, Walder R, Garzaro D (1997) Anti-HIV activity of extracts from Calendula officinalis flowers. Biomedicine and Pharmacotherapy 51(4):176180.

Kammoun M, Koubaa I, Ben Ali Y, Jarraya R, Gargouri Y, Damak M, Bezzine S (2010) Inhibition of pro-inflammatory secreted phospholipase A2 by extracts from Cynara cardunculus L. Applied Biochemistry and Biotechnology 162(3):662-670.

Kappel VD, Pereira DF, Cazarolli LH, Guesser SM, da Silva CH, Schenkel EP, Reginatto FH, Silva FR (2012) Short and long-term effects of Baccharis articulata on glucose homeostasis. Molecules 17(6):6754-6768.

Katinas L, Gutiérrez DG, Grossi MA, Crisci JV (2007) Panorama de la familia Asteraceae (= Compositae) en la República Argentina. Boletín Sociedad Argentina de Botánica 42(1-2):113-129. 
Khan AU, Gilani AH (2009) Antispasmodic and bronchodilator activities of Artemisia vulgaris are mediated through dual blockade of muscarinic receptors and calcium influx. Journal of Ethnopharmacology 126:480-486.

Khan AU, Gilani AH (2011) Blood pressure lowering, cardiovascular inhibitory and bronchodilatory actions of Achillea millefolium. Phytotherapy Research 25(4):577-583.

Kim JW, Han JY, Hong JT, Li R, Eun JS, Oh KW (2011) Ethanol extract of the flower Chrysanthemum morifolium augments pentobarbital-induced sleep behaviors: Involvement of $\mathbf{C l}$ channel activation. Evidence Based Complementary and Alternative Medicine, doi: 10.1155/2011/109164.

Kokoska L, Polesny Z, Rada V, Nepovim A, Vanek T (2002) Screening of some Siberian medicinal plants for antimicrobial activity. Journal of Ethnopharmacology 82(1):51-53.

König GM, Wright AD, Keller WJ, Judd RL, Bates S, Day C (1998) Hypoglycaemic activity of an HMG-containing flavonoid glucoside, chamaemeloside, from Chamaemelum nobile. Planta Medica 64(7):612-614.

Koo H, Hong S, Song B, Kim C, Kim H (2004) Taraxacum officinale induces cytotoxicity through TNF- $\alpha$ and IL-1 $\alpha$ secretion in Hep G2 cells. Life Sciences 74(9):1149-1157.

Krecman V, Skottova N, Walterova D, Ulrichova J, Simanek V (1998) Silymarin inhibits the development of diet-induced hypercholesterolemia in rats. Planta Medica 64:138-142.

Ladio $\mathrm{AH}$, Molares $\mathrm{S}$ (2010) Aspectos do estudo da dinâmica do uso de produtos etnobiológicos não tradicionais. In Albuquerque UP, Lucena RF, Cunha L (eds), Métodos e Técnicas na Pesquisa Etnobiológica e Etnoecológica, NUPEEA, Recife, pp. 267- 278.

Lahlou S, Tangi K, Lyoussi B, Morel N (2008) Vascular effects of Tanacetum vulgare L. leaf extract: in vitro pharmacological study. Journal of Ethnopharmacology 120(1):98-102.

Lavelli V (2008) Antioxidant activity of processed red chicory (Cichorium intybus) evaluated in xanthine oxidase, myeloperoxidase and diaphorase-catalyzed reactions. Journal of Agricultural and Food Chemistry 56:7194-7200.

Lee BR, Lee JH, An HJ (2012) Effects of Taraxacum officinale on fatigue and immunological parameters in mice. Molecules 17(11):13253-13265.

Lee JS, Kim HJ, Lee YS (2003) A new anti-HIV flavonoid glucuronide from Chrysanthemum morifolium. Planta Medica 69(9):859-861.

Lee YJ, Choi DH, Cho GH, Kim JS, Kang DG, Lee HS (2012) Arctium lappa ameliorates endothelial dysfunction in rats fed with high fat/cholesterol diets. BMC Complementary and Alternative Medicine 6,12:116, doi: 10.1186/1472-6882-12-116.

Li Y, Zhang Y, Fu M, Yao Q, Zhuo H, Lu Q, Niu X, Zhang P, Pei Y, Zhang K (2012) Parthenolide induces apoptosis and lytic cytotoxicity in Epstein-Barr virus-positive Burkitt lymphoma. Molecular Medicine Report. 2012, doi: 10.3892/mmr.2012.959.

Li YP, Wang YM (1988) Evaluation of tussilagone: A cardiovascular-respiratory stimulant isolated from Chinese herbal medicine. General Pharmacology 19(2):261-263.

Li ZY, Zhi HJ, Zhang FS, Sun HF, Zhang LZ, Jia JP, Xing J, Qin XM (2013) Metabolomic profiling of the antitussive and expectorant plant Tussilago 
farfara by nuclear magnetic resonance spectroscopy and multivariate data analysis. Journal of Pharmaceutical Biomedical Analysis 75:158-164.

Lii CK, Lei YP, Yao H, Hsieh YS, Tsai C, Liu KL, Chen HW (2010) Chrysanthemum morifolium reduces the oxidized LDL-induced expression of intercellular adhesion molecule-1 and E-selectin in human umbilical vein endothelial cells. Journal of Ethnopharmacology 128(1):213-220.

Lima GM, Bonfim RR, Silva MR, Thomazzi SM, Santos MR, Quintans-Júnior LJ, Bonjardim LR, Araújo AAS (2011) Assessment of antinociceptive and antiinflammatory activities of Porophyllum ruderale, Asteraceae, aqueous extract. Revista Brasileira de Farmacognosia 21(3):486-490.

Lin GH, Lin L, Liang HW, Ma X, Wang JY, Wu LP, Jiang HD, Bruce IC, Xia Q (2010) Antioxidant action of a Chrysanthemum morifolium extract protects rat brain against ischemia and reperfusion injury. Journal of Medicinal Food 13(2):306-311.

Liu J, Kao P, Chan P, Hsu G, Hou C, Lien G, Hsieh M, Chen Y, Cheng J (2003) Mechanism of the antihypertensive effect of stevioside in anesthetized dogs. Pharmacology 67:14-20.

Lizarraga E, Castro F, Fernández F, de Lampasona MP, Catalán CA (2012) Antioxidant, hemolytic and cytotoxic activities of Senecio species used in traditional medicine of Northwestern Argentina. Natural Product Communications 7(5):607-608.

Lobo AR, Colli C, Alvares EP, Filisetti TM (2007) Effects of fructans-containing yacon (Smallanthus sonchifolius) flour on caecum mucosal morphometry, calcium and magnesium balance, and bone calcium retention in growing rats. British Journal of Nutrition 97:776-85.

Lopes-Lutz D, Alviano DS, Alviano CS, Kolodziejczyk PP (2008) Screening of chemical composition, antimicrobial and antioxidant activities of Artemisia essential oils. Phytochemistry 69(8):1732-1738.

Machado FB, Yamamoto RE, Zanoli K, Nocchi SR, Novello CR, Schuquel IT, Sakuragui C, Luftmann H, Ueda-Nakamura T, Nakamura C, Mello JC (2012) Evaluation of the antiproliferative activity of the leaves from Arctium lappa by a bioassay-guided fractionation. Molecules 17(2):1852-1859.

Macía MJ, García E, Vidaurre PJ (2005) An ethnobotanical survey of medicinal plants commercialized in the markets of La Paz and El Alto, Bolivia. Journal of Ethnopharmacology 97(2):337-350.

Mahesh A, Jeyachandran R, Cindrella L, Thangadurai D, Veerapur VP, Muralidhara Rao D (2010) Hepatocurative potential of sesquiterpene lactones of Taraxacum officinale on carbon tetrachloride induced liver toxicity in mice. Acta Biologica Hungarica 61(2):175-190.

Mangiaterra PA (2005) Evaluación de parámetros botánicos y fitoquímicos para el control de calidad de «carquejas». Tesina 126: 1-46. Universidad de Belgrano, Buenos Aires.

Martijena ID, Garcia DA, Marin RH, Perillo MA, Zygadlo JP (1998) Anxiogenic-like and antidepressant-like effects of the essential oil from Tagetes minuta. Fitoterapia 69(2):155-160.

Martin GJ (2004) Ethnobotany. A methods manual. Earthscan. London.

Martínez SF (2008) La cognición corporizada en prácticas: implicaciones para la filosofía de la ciencia. In: Martínez SF, Huang X, Guillaumin G. (eds) Historia, prácticas y estilos en la filosofía de la ciencia: hacia una epistemología plural. UNAM, México, pp. 212-231. 
Martínez Crovetto R (1981) Las plantas utilizadas en medicina popular en el Noroeste de Corrientes (Argentina). Instituto M. Lillo, Tucumán.

Mascolo N, Autore G, Capasso, F. Menghini A, Fasulo MP (1987) Biological screening of Italian medicinal plants for anti-inflammatory activity. Phytotherapy Research 1(1):28-31.

Mathema V, Koh Y, Thakuri B, Sillanpää M (2012) Parthenolide, a sesquiterpene lactone, expresses multiple anti-cancer and anti-inflammatory activities. Inflammation 35(2):560-565.

Matić IZ, Juranić Z, Savikin K, Zdunić G, Nađvinski N, Gođevac D (2012) Chamomile and marigold tea: Chemical characterization and evaluation of anticancer activity. Phytotherapy Research 2012, doi: 10.1002/ptr.4807.

Mats MN, Bogatkina VF, Vishnyakov Yu S (1987) Contraceptive activity of an extract from Ambrosia artemisiifolia. Rastitel'nye Resursy 23:125-129.

Mayhua G, Suárez S, del Castillo T (2013). Capacidad antioxidante in vitro y contenido de polifenoles y flavonoides en el extracto hidroalcóholico de las hojas de Trixis divaricata (hank'u chuta). Anales de la Facultad de Medicina (Lima) 73(1):S37.

Meneses R, Ocazionez RE, Martínez JR, Stashenko EE (2009) Inhibitory effect of essential oils obtained from plants grown in Colombia on yellow fever virus replication in vitro. Annals of Clinical Microbiology and Antimicrobials 8:8, doi: 10.1186/1476-0711-8-8.

Metwally NS, Kholeif TE, Ghanem KZ, Farrag AR, Ammar NM, Abdel-Hamid AH (2011) The protective effects of fish oil and artichoke on hepatocellular carcinoma in rats. European Review for Medical and Pharmacological Sciences 15(12):1429-1444.

Mileo AM, Di Venere D, Linsalata V, Fraioli R, Miccadei S (2012) Artichoke polyphenols induce apoptosis and decrease the invasive potential of the human breast cancer cell line MDA-MB231. Journal of Cellular Physiology 227(9):3301-3309.

Minaiyan M, Ghannadi AR, Mahzouni P, Abed AR (2012) Preventive effect of Cichorium Intybus L. two extracts on cerulein-induced acute pancreatitis in mice. International Journal of Preventive Medicine 3(5):351-357.

Miño J, Muschietti L, Ferraro G, Martino V, Acevedo C (2005) Antinociceptive activity of Eupatorium buniifolium aqueous extract. Fitoterapia 76(1):100103.

Miño J, Muschietti L, Ferraro G, Martino V, Acevedo C (2007) Neuropharmacological activity of Eupatorium buniifolium aqueous extract in mice. Phytotherapy Research 21(4):362-365.

Mirandola L, Justo GZ, Queiroz ML (2002) Modulation by Acanthospermum australe extracts of the tumor induced hematopoietic changes in mice. Immunopharmacology and Immunotoxicology 24(2):275-288.

Mishima S, Saito K, Maruyama H, Inoue M, Yamashita T, Ishida T, Gu Y (2004) Antioxidant and immuno-enhancing effects of Echinacea purpurea. Biological and Pharmaceutical Bulletin 27(7):1004-1009.

Modaresi M, Resalatpour N (2012) The effect of Taraxacum officinale hydroalcoholic extract on blood cells in mice. Advances in Hematology 2012, doi: 10.1155/2012/653412.

Morales G, Sierra P, Mancill A, Paredes A, Loyola L, Gallardo O, Borquez J (2003) Secondary metabolites from four medicinal plants from northern Chile: 
antimicrobial activity and biotoxicity against Artemia salina. Journal of the Chilean Chemical Society 48:13-18.

Morales G, Paredes A, Sierra P, Loyola L (2009) Cytotoxicity, scavenging and lipid peroxidation-inhibiting activities of 5,3',4'-trihydroxy-7methoxyflavanone isolated from Haplopappus rigidus. Journal of the Chilean Chemical Society 54(2):105-107.

Moura NA, Caetano BF, Sivieri K, Urbano LH, Cabello C, Rodrigues MA, Barbisan LF (2012) Protective effects of yacon intake on experimental colon carcinogenesis. Food and Chemical Toxicology 50(8):2902-2910.

Muschietti L, Gorzalczany S, Ferraro G, Acevedo C, Martino V (2001) Phenolic compounds with anti-inflammatory activity from Eupatorium buniifolium. Planta Medica 67(8):743-744.

Muschietti L, Derita M, Sülsen V, de Dios Muñoz J, Ferraro G, Zacchino S, Martino V (2005) In vitro antifungal assay of traditional Argentine medicinal plants. Journal of Ethnopharmacology 102(2):233-238.

Muthaura C, Rukunga G, Chhabra S, Omar S, Guantai A, Mwitari P, Mungai G, Njagi $\mathrm{N}$ (2007) Antimalarial activity of some plants traditionally used in Meru district of Kenya. Phytotherapy Research 21(9):860-867.

Nahas R, Balla A (2011). Complementary and alternative medicine for prevention and treatment of the common cold. Canadian Family Physician 57(1):31-36.

Nayak BS, Raju SS, Rao AV (2007) Wound healing activity of Matricaria recutita extract. Journal of Wound Care 16(7):298-302.

Nchu F, Magano SR, Eloff JN (2012) In vitro anti-tick properties of the essential oil of Tagetes minuta (Asteraceae) on Hyalomma rufipes (Acari: Ixodidae). Onderstepoort Journal of Veterinary Research 79(1):E1-E5.

Nostro A, Cannatelli MA, Marino A, Picerno I, Pizzimenti FC, Scoglio ME, Spataro P (2003) Evaluation of antiherpesvirus-1 and genotoxic activities of Helichrysum italicum extract. New Microbiologica 26(1):125-128.

Obolskiy D, Pischel I, Feistel B, Glotov N, Heinrich M (2011) Artemisia dracunculus (tarragon): A critical review of its traditional use, chemical composition, pharmacology, and safety. Journal of Agricultural and Food Chemistry 59(21):11367-11384.

Oliveira ABS, Dias Filho BP, Nakamura CV, Ueda-Nakamura T (2009) Antiviral activity and mode of action of a peptide isolated from Helianthus annus. Planta Medica 75, doi: 10.1055/s-0029-1234650.

Oliveira AC, Endringer DC, Amorim LA, das Graças L Brandão M, Coelho MM (2005) Effect of the extracts and fractions of Baccharis trimera and Syzygium cumini on glycaemia of diabetic and non-diabetic mice. Journal of Ethnopharmacology 102(3):465-469.

Oliveira AJ, Cordeiro LM, Gonçalves RA, Ceole LF, Ueda-Nakamura T, lacomini M (2013) Structure and antiviral activity of arabinogalactan with (1-6)- $\beta$-dgalactan core from Stevia rebaudiana leaves. Carbohydrate Polymers 94(1):179-184.

Önal S, Timur S, Okutucu B, Zihnioğlu F (2005) Inhibition of $\alpha$-glucosidase by aqueous extracts of some potent antidiabetic medicinal herbs. Preparative Biochemistry and Biotechnology 35(1):29-36.

Ono M, Masuoka C, Odake Y, Ikegashira S, Ito Y, Nohara T (2000) Antioxidative constituents from Tessaria integrifolia. Food Science and Technology Research 6(2):106-114. 
Orekhov AN (2013) Direct anti-atherosclerotic therapy; Development of natural anti-atherosclerotic drugs preventing cellular cholesterol retention. Current Pharmaceutical Design 2013, PMID 23438951.

Pádua C, Silva LD, Rossoni Júnior JV, Humberto JL, Chaves MM, Silva ME, Pedrosa ML, Costa DC (2010) Antioxidant properties of Baccharis trimera in the neutrophils of Fisher rats. Journal of Ethnopharmacology 129(3):381-386.

Pareek A, Suthar M, Rathore G, Bansal V (2011) Feverfew (Tanacetum parthenium): A systematic review. Pharmacognosy Reviews 5(9):103-110.

Pastor A, Zelada BR (2006). Estudio fitoquímico de Flaveria bidentis (L.) Kuntze (Asteraceae). Revista de la Sociedad Química del Perú 72(1):3-11.

Paul EL, Lunardelli A, Caberlon E, de Oliveira CB, Santos RC, Biolchi V, Bastos CM, Moreira KB, Nunes FB, Gosmann G, de Oliveira JR (2009) Anti-inflammatory and immunomodulatory effects of Baccharis trimera aqueous extract on induced pleurisy in rats and lymphoproliferation in vitro. Inflammation 32(6):419-425.

Pedreschi R, Campos D, Noratto G, Chirinos R, Cisneros-Zevallos L (2003) Andean yacon root fructooligosaccharides as a potential novel source of prebiotics. Journal of Agricultural and Food Chemistry 51(18):5278-5284.

Peluso G, De Feo V, De Simone F, Bresciano E, Vuotto M (1995) Studies on the inhibitory effects of caffeoylquinic acids on monocyte migration and superoxide ion production. Journal of Natural Products 58(5):639-646.

Pereira, FE, Figueiredo Guimaraes, L, Cerutti SM, Oliveira Rodrigues RF, Pulz Araujo CE (2005). Análisis preliminar antiulcerogénico y químico de las partes aéreas de Trixis divaricata Spreng. Acta Farmacéutica Bonaerense 24(1) 80-84.

Pérez C, Agnese AM, Cabrera JL (1999) The essential oil of Senecio graveolens (Compositae): Chemical composition and antimicrobial activity tests. Journal of Ethnopharmacology 66(1):91-96.

Pérez-García F, Marín E, Cañigueral S, Adzet T (1996) Anti-inflammatory action of Pluchea sagittalis: Involvement of an antioxidant mechanism. Life Sciences 59(24):2033-2040.

Petenatti EM, Petenatti ME, Zallocco Y, Del Vitto, LA. (2004a) Control de calidad de medicamentos herbarios en el Centro-oeste Argentino. III. Gaillardia megapotamica (Asteraceae: Helenieae), «topasaire» o «botón de oro». Acta Farmacéutica Bonaerense 23(4):442-447.

Petenatti EM, Nievas CM, Petenatti ME, Del Vitto LA (2004b) Medicamentos Herbarios en el Centro-oeste Argentino. IV. «Marcelas» y «vira-viras» en muestras comerciales. Acta Farmacéutica Bonaerense 23(4):484-491.

Pieroni A, Muenz H, Akbulut M, Başer K, Durmuşkahya C (2005) Traditional phytotherapy and trans-cultural pharmacy among Turkish migrants living in Cologne, Germany. Journal of Ethnopharmacology 102(1):69-88.

Pires J, Mendes F, Negri G, Duarte-Almeida J, Carlini E (2009) Antinociceptive peripheral effect of Achillea millefolium and Artemisia vulgaris: Both plants known popularly by brand names of analgesic drugs. Phytotherapy Research 23(2):212-219.

Pisha E, Chai H, Lee I, Chagwedera T, Farnsworth N, Cordell G, Beecher C, Fong $H$, Kinghorn A, Brown D, Wani M, Wall M, Hieken T, Das Gupta T, Pezzuto J (1995) Discovery of betulinic acid as a selective inhibitor of human melanoma that functions by induction of apoptosis. Nature Medicine 10:1046-1051. 
Pleschka S, Stein M, Schoop R, Hudson JB (2009) Anti-viral properties and mode of action of standardized Echinacea purpurea extract against highly pathogenic avian influenza virus (H5N1, H7N7) and swine-origin H1N1 (SOIV). Virology Journal 6:197, doi: 10.1186/1743-422X-6-197.

Plohmann B, Bader G, Hiller K, Franz G (1997) Immunomodulatory and antitumoral effects of triterpenoid saponins. Pharmazie 52(12): 953-957.

Pochettino ML, Arenas PM, Sánchez D, Correa R (2008) Conocimiento botánico tradicional, circulación comercial y consumo de plantas medicinales en un área urbana de Argentina. Boletín Latinoamericano y del Caribe de Plantas Medicinales y Aromáticas 7(3):141-148.

Pochettino ML, Puentes JP, Buet-Costantino F, Arenas PM, Ulibarri EA, Hurrell JA (2012a) Functional foods and nutraceuticals in a market of Bolivian inmigrants in Buenos Aires (Argentina). Evidence-Based Complementary and Alternative Medicine, doi:10.1155/2012/320193.

Pochettino ML, Bonicatto M, Buet Costantino F, Hurrell JA (2012b). La horticultura en un contexto periurbano: estudio comparativo entre huertos familiares y productivos en la provincia de Buenos Aires (Argentina). Memorias II Congreso Boliviano de Botánica-III Congreso Latinoamericano de Etnobiología-I Simposio Boliviano de Etnobotánica, 124. La Paz.

Poiată A, Tuchiluş C, Ivănescu B, lonescu A, Lazăr MI (2009) Antibacterial activity of some Artemisia species extract. Revista Medico-Chirurgicala a Societatii de Medici si Naturalisti din lasi 113(3):911-914.

Polatoglu K, Demirci F, Demirci B, Gören N, Başer K (2010) Antibacterial activity and the variation of Tanacetum parthenium essential oils from Turkey. Journal of Oleo Science 59(4):177-184.

Portillo A, Vila R, Freixa B, Adzet T, Cañigueral S (2001) Antifungal activity of Paraguayan plants used in traditional medicine. Journal of Ethnopharmacology 76(1):93-98.

Preethi K, Kuttan R (2009) Hepato and reno-protective action of Calendula officinalis flower extract. Indian Journal of Experimental Biology 47:163-168.

Preethi K, Kuttan G, Kuttan R (2009) Anti-inflammatory activity of flower extract of Calendula officinalis and mechanism of action. Indian Journal of Experimental Biology 47:113-120.

Pushparaj P, Low H, Manikandan J, Tan B, Tan C (2007) Anti-diabetic effects of Cichorium intybus in streptozotocin-induced diabetic rats. Journal of Ethnopharmacology 111(2):430-434.

Ranilla LG, Kwon YI, Apostolidis E, Shetty K (2010) Phenolic compounds, antioxidant activity and in vitro inhibitory potential against key enzymes relevant for hyperglycemia and hypertension of commonly used medicinal plants, herbs and spices in Latin America. Bioresource Technology 101(12):4676-4689.

Ranpariya VL, Parmar SK, Sheth NR, Chandrashekhar VM (2011) Neuroprotective activity of Matricaria recutita against fluoride-induced stress in rats. Pharmaceutical Biology 49(7):696-701.

Ribnicky DM, Poulev A, Watford M, Cefalu WT, Raskin I (2006) Antihyperglycemic activity of Tarralin, an ethanolic extract of Artemisia dracunculus L. Phytomedicine 13(8):550-557.

Ríos JL (2008) Helichrysum italicum: una planta mediterránea con potencial terapéutico. Revista de Fitoterapia 8(1):13-20. 
Rocha Martins LR, Brenzan MA, Nakamura CV, Dias Filho BP, Nakamura TU, Ranieri Cortez LE, Garcia Cortez DA (2011) In vitro antiviral activity from Acanthospermum australe on herpesvirus and poliovirus. Pharmaceutical Biology 49(1):26-31.

Rodrigo GC, Almanza GR, Akesson B, Duan RD (2010) Antiproliferative activity of extracts of some Bolivian medicinal plants. Journal of Medicinal Plants Research 4(21):2204-2210.

Romio E, Gurni, A (2007) Estudio micrográfico preliminar de las estructuras foliares de dos especies palustres americanas con potencial actividad antiviral. Boletín Latinoamericano y del Caribe de Plantas Medicinales y Aromáticas 6(5):219-220.

Rondina RDV, Bandoni AL, Coussio JD (2008) Especies medicinales argentinas con potencial actividad analgésica. Dominguezia 24(1):47-69.

Rosella MA, Rojas Durán R, Aquila SV, del Valle ME, Spegazzini D, Debenedetti S (2010) Actividad antimicobacteriana in vitro de Gaillardia cabrerae y G. megapotamica var. scabiosioides (Asteraceae). Latin American Journal of Pharmacy 29:816-819.

Rossi T, Melegari M, Bianchi A, Albasini A, Vampa G (1988) Sedative, antiinflammatory and anti-diuretic effects induced in rats by essential oils of varieties of Anthemis nobilis: A comparative study. Pharmacological Research Communications 20(Suppl 5):71-74.

Ruffa MJ, Ferraro G, Wagner ML, Calcagno ML, Campos RH, Cavallaro L (2002) Cytotoxic effect of Argentine medicinal plant extracts on human hepatocellular carcinoma cell line. Journal of Ethnopharmacology 79(3):335-339.

Sabini MC, Escobar FM, Tonn CE, Zanon SM, Contigiani MS, Sabini LI (2012) Evaluation of antiviral activity of aqueous extracts from Achyrocline satureioides. Natural Products Research 26(5):405-415.

Saini S, Sharma, S (2011) Helianthus annuus (Asteraceae): A review. International Journal of Pharma Professional's Research 2(4):465-470.

Sala A, Recio M, Giner RM, Máñez S, Tournier H, Schinella G, Ríos JL (2002) Antiinflammatory and antioxidant properties of Helichrysum italicum. Journal of Pharmacy and Pharmacology 54(3):365-371.

Salama $\mathrm{RH}$ (2012) Matricaria chamomilla attenuates cisplatin nephrotoxicity. Saudi Journal of Kidney Diseases and Transplantation 23(4):765-772.

Salazar W, Cárdenas J, Nuñez M, Fernández I, Villegas L, Pacheco L, Untiveros G (2007) Estudio fitoquímico y de la actividad antihelmíntica de los extractos de Euphorbia huanchahana y Baccharis salicifolia. Revista de la Sociedad Química de Perú 73(3):150-157.

Sandhu DS, Heinrich M (2005) The use of health foods, spices and other botanicals in the Sikh community in London. Phytotherapy Research 19(7):633-642.

Santin JR, Lemos M, Klein Júnior LC, Niero R, de Andrade SF (2010) Antiulcer effects of Achyrocline satureoides (Lam.) DC. (Asteraceae), a folk medicine plant, in different experimental models. Journal of Ethnopharmacology 130(2):334-339.

Sarris J, Panossian A, Schweitzer I, Stough C, Scholey A (2011) Herbal medicine for depression, anxiety and insomnia: a review of psychopharmacology and clinical evidence. European Neuropsychopharmacology 21(12):841-860. 
Sayyah M, Nadjafnia L, Kamalinejad M (2004) Anticonvulsant activity and chemical composition of Artemisia dracunculus essential oil. Journal of Ethnopharmacology 94(2-3):283-287.

Schmidt C, Fronza M, Goettert M, Geller F, Luik S, Flores EM, Bittencourt CF, Zanetti GD, Heinzmann BM, Laufer S, Merfort I (2009) Biological studies on Brazilian plants used in wound healing. Journal of Ethnopharmacology 122(3):523-532.

Setzer WN (2009) Essential oils and anxiolytic aromatherapy. Natural Product Communications 4(9):1305-1316.

Shafi G, Hasan TN, Syed NA, Al-Hazzani A, Alshatwi AA, Jyothi A, Munshi A (2012) Artemisia absinthium: A novel potential complementary and alternative medicine for breast cancer. Molecular Biology Reports 39(7):7373-7379.

Shahwar D, Raza MA, Saeed A, Riasat M, Chattha FI, Javaid M, Ullah S, Ullah S (2012) Antioxidant potential of the extracts of Putranjiva roxburghii, Conyza bonariensis, Woodfordia fruiticosa and Senecio chrysanthemoids. African Journal of Biotechnology 11(18):4288-4295.

Shaker E, Mahmoud H, Mnaa S (2010) Silymarin, the antioxidant component and Silybum marianum extracts prevent liver damage. Food and Chemical Toxicology 48(3):803-806.

Sharifi R, Pasalar P, Kamalinejad M, Dehpour AR, Tavangar SM, Paknejad M, Mehrabani Natanzi M, Nourbakhsh M, Ahmadi Ashtiani HR, Akbari M, Rastegar H (2013) The effect of silymarin (Silybum marianum) on human skin fibroblasts in an in vitro wound healing model. Pharmaceutical Biology 51(3):298-303.

Shikov A, Pozharitskaya O, Makarov V, Kvetnaya A (2008) Antibacterial activity of Chamomilla recutita. Phytotherapy Research 22(2):252-253.

Shivanna N, Naika M, Khanum F, Kaul VK (2013) Antioxidant, antidiabetic and renal protective properties of Stevia rebaudiana. Journal of Diabetes and its Complications 27(2):103-113.

Shukla S, Mehta A, Bajpai V, Shukla S (2009) In vitro antioxidant activity and total phenolic content of ethanolic leaf extract of Stevia rebaudiana. Food and Chemical Toxicology 47(9):2338-2343.

Sigstedt SC, Hooten CJ, Callewaert MC, Jenkins AR, Romero AE, Pullin MJ, Kornienko A, Lowrey TK, Slambrouck SV, Steelant WF (2008) Evaluation of aqueous extracts of Taraxacum officinale on growth and invasion of breast and prostate cancer cells. International Journal of Oncology 32(5):1085-1090.

Silva LM da, Allemand A, Mendes DA, Dos Santos AC, André E, de Souza LM, Cipriani TR, Dartora N, Marques MC, Baggio CH, Werner MF (2013) Ethanolic extract of roots from Arctium lappa $\mathrm{L}$. accelerates the healing of acetic acid-induced gastric ulcer in rats: Involvement of the antioxidant system. Food and Chemical Toxicology 51:179-187.

Simoniato E, Ilha V, Mallmann A, Porto C, Dalcol I, Morel A (2008) Chemical composition and antimicrobial activity of the volatile oil from Baccharis articulate (Lam.) Pers. Journal of Essential Oil Research 20(4):266-368.

Skaudickas D, Kondrotas A, Kevelaitis EC, Venskutonis P (2009) The effect of Echinacea purpurea extract on experimental prostate hyperplasia. Phytotherapy Research 23(10):1474-1478. 
Smirnova G, Samoilova Z, Muzyka N, Oktyabrsky O (2012) Influence of plant polyphenols and medicinal plant extracts on antibiotic susceptibility of Escherichia coli. Journal of Applied Microbiology 113(1):192-199.

Song FL, Gan RY, Zhang Y, Qin X, Kuan L, Li HB (2010) Total phenolic contents and antioxidant capacities of selected chinese medicinal plants. International Journal of Molecular Sciences 11(6): 2362-2372.

Sorarú S, Bandoni A (1978) Plantas de la medicina popular argentina. Albatros, Buenos Aires.

Soria EA, Goleniowski ME, Cantero JJ, Bongiovanni GA (2008) Antioxidant activity of different extracts of Argentinian medicinal plants against arsenicinduced toxicity in renal cells. Human and Experimental Toxicology 27(4):341-346.

Sosa A, Fusco M, Petenatti ME, Juárez A, Del Vitto LA, Petenatti EM (2007) Estudios farmacognósticos y farmacológicos comparativos sobre tres especies diuréticas de amplio uso popular en el Centro-oeste argentino. Boletín Latinoamericano y del Caribe de Plantas Medicinales y Aromáticas 6(6):386-338.

Sosa A, Fusco M, Rossomando P, Juárez A, Robles S, Petenatti EM, Pelzer L (2011) Anti-inflammatory properties from isolated compounds of Cyclolepis genistoides. Pharmaceutical Biology 49(7):675-678.

Souza G de, Haas A, von Poser G, Schapoval E, Elisabetsky E (2004) Ethnopharmacological studies of antimicrobial remedies in the south of Brazil. Journal of Ethnopharmacology 90(1):135-143.

Souza MC, Siani AC, Ramos MF, Menezes-de-Lima OJ, Henriques MG (2003) Evaluation of anti-inflammatory activity of essential oils from two Asteraceae species. Pharmazie 58(8):582-586.

Souza P, Gasparotto A, Crestani S, Stefanello M, Marques M, da Silva-Santos J, Kassuya C (2011) Hypotensive mechanism of the extracts and artemetin isolated from Achillea millefolium (Asteraceae) in rats. Phytomedicine 18(10):819-825.

Spjut RW (2005) Relationships between plant folklore and antitumor activity. An historical review. Sida 21(4):2205-2241.

Srivastava J, Gupta S (2007) Antiproliferative and apoptotic effects of chamomile extract in various human cancer cells. Journal of Agricultural and Food Chemistry 55(23):9470-9478.

Stanciuc AM, Gaspar A, Moldovan L, Saviuc C, Popa M, Măruţescu L (2011) In vitro antimicrobial activity of Romanian medicinal plants hydroalcoholic extracts on planktonic and adhered cells. Roumanian Archives of Microbiology and Immunology 70(1):11-14.

Stanojević L, Stanković M, Nikolić V, Nikolić L, Ristić D, Canadanovic-Brunet J, Tumbas $\mathrm{V}$ (2009) Antioxidant activity and total phenolic and flavonoid contents of Hieracium pilosella L. extracts. Sensors (Basel) 9(7):5702-5714.

Steenkamp V, Gouws MC (2006) Cytotoxicity of six South African medicinal plant extracts used in the treatment of cancer. South African Journal of Botany 72(4):630-633.

Stevens PF (2013) Angiosperm Phylogeny Website, version 12 [<http://www.mobot.org/MOBOT/research/APweb/] Accessed 15 April 2013.

Subashini R, Rakshitha SU (2012) Phytochemical screening, antimicrobial activity and in vitro antioxidant investigation of methanolic extract of seeds from Helianthus annuus L. Chemical Sciences Review Letters 1(1):30-34. 
Sülsen VP, Cazorla SI, Frank FM, Redko FC, Anesini CA, Coussio JD, Malchiodi EL, Martino VS, Muschietti LV (2007) Trypanocidal and leishmanicidal activities of flavonoids from Argentine medicinal plants. The American Journal of Tropical Medicine and Hygiene 77(4):654-659.

Szakiel A, Ruszkowski D, Grudniak A, Kurek A, Wolska K, Doligalska M, Janiszowska W (2008) Antibacterial and antiparasitic activity of oleanolic acid and its glycosides isolated from Calendula officinalis. Planta Medica 74(14):17091715.

Takahashi HT, Britta EA, Longhini R, Ueda-Nakamura T, Palazzo de Mello JC, Nakamura CV (2013) Antileishmanial activity of 5-Methyl-2,2':5',2"terthiophene Isolated from Porophyllum ruderale is related to mitochondrial dysfunction in Leishmania amazonensis. Planta Medica 79(5):330-333.

Takaki M, de Campos T, Santana G (1985) Antimicrobial activity in leaves extracts of Stevia rebaudiana. Revista do Instituto Universitário de Pernambuco 22:33-39.

Tamayo C, Richardson MA, Diamond S, Skoda I (2000) The chemistry and biological activity of herbs used in Flor-Essence herbal tonic and Essiac. Phytotherapy Research 14:1-14.

Tariq KA, Chishti MZ, Ahmad F, Shawl AS (2009) Anthelmintic activity of extracts of Artemisia absinthium against ovine nematodes. Veterinary Parasitology 160(1-2):83-88.

Temraz A, El-Tantawy WH (2008) Characterization of antioxidant activity of extract from Artemisia vulgaris. Pakistan Journal of Pharmaceutical Sciences 21(4):321-326.

Tereschuk ML, Riera MV, Castro GR, Abdala LR (1997) Antimicrobial activity of flavonoids from leaves of Tagetes minuta. Journal of Ethnopharmacology 56(3):227-232.

Tigno XT, Gumila E (2000) In vivo microvascular actions of Artemisia vulgaris L. in a model of ischemia-reperfusion injury in the rat intestinal mesentery. Clinical Hemorheology and Microcirculation 23(2-4):159-165.

Tognolini M, Barocelli E, Ballabeni V, Bruni R, Bianchi A, Chiavarini M, Impicciatore $M(2006)$ Comparative screening of plant essential oils: Phenylpropanoid moiety as basic core for antiplatelet activity. Life Sciences 78(13):1419-32

Torres CV, Domínguez MJ, Carbonari JL, Sabini MC, Sabini LI, Zanon SM (2011) Study of antiviral and virucidal activities of aqueous extract of Baccharis articulata against Herpes virus. Natural Product Communications 6(7):993994.

Tsai YL, Chiu C, Yi-Fu Chen J, Chan K, Lin S (2012) Cytotoxic effects of Echinacea purpurea flower extracts and cichoric acid on human colon cancer cells through induction of apoptosis. Journal of Ethnopharmacology 143(3):914919.

Turgut F, Bayrak O, Catal F, Bayrak R, Atmaca AF, Koc A, Akbas A, Akcay A, Unal D (2008) Antioxidant and protective effects of silymarin on ischemia and reperfusion injury in the kidney tissues of rats. International Urology and Nephrology 40(2):453-460.

Ukiya M, Akihisa T, Yasukawa K, Kasahara Y, Kimura Y, Koike K, Nikaido T, Takido M (2001) Constituents of Compositae plants. 2. Triterpene diols, triols, and their 3-o-fatty acid esters from edible Chrysanthemum flower extract 
Hurrell and Puentes 2013. Medicinal and aromatic species of Asteraceae commercialized in the conurbation Buenos Aires-La Plata (Argentina). Ethnobio Conserv 2:7

and their anti-inflammatory effects. Journal of Agricultural and Food Chemistry 49(7):3187-3197.

Valentová K, Moncion A, Waziers I, Ulrichová J (2004) The effect of Smallanthus sonchifolius leaf extracts on rat hepatic metabolism. Cell Biology and Toxicology 20(2):109-120.

Vanaclocha B, Cañigueral S. 2003. Fitoterapia: vademécum de prescripción. Masson, Barcelona.

Varela F (1990) Conocer.1 ed. Gedisa, Barcelona.

Vasudeva N, Yadav N, Sharma SK (2012) Natural products: A safest approach for obesity. Chinese Journal of Integrative Medicine 18(6):473-80.

Verástegui MA, Sánchez CA, Heredia NL, García-Alvarado JS (1996) Antimicrobial activity of extracts of three major plants from the Chihuahuan desert. Journal of Ethnopharmacology 52(3):175-177.

Verdi LG, Brighente IMC, Pizzolatti MG (2005) Genero Baccharis (Asteraceae): aspectos químicos, econômicos e biológicos. Química Nova 28(1):85-94.

Vitalini S, Beretta G, Iriti M, Orsenigo S, Basilico N, Dall'Acqua S, Iorizzi M, Fico G (2011) Phenolic compounds from Achillea millefolium and their bioactivity. Acta Biochimica Polonica 58(2):203-209.

Vivot E, Massa R, Cruañes MJ, Muñoz J, Ferraro G, Gutkind G, Martino V (2007) Actividad antimicrobiana in vitro de seis especies autóctonas de Entre Ríos (Argentina). Latin American Journal of Pharmacy 26(4):563-566.

Volpato G, Godínez, D, Beyra A (2009) Migration and ethnobotanical practices. The case of Tifey among Haitian immigrants in Cuba. Human Ecology 37(1):43-53.

Voon HC, Bhat R, Rusul G (2012) Flower extracts and their essential oils as potential antimicrobial agents for food uses and pharmaceutical applications. Comprehensive Reviews in Food Science and Food Safety 11:34-55.

Wachsman M, Coto E, Martino V (1988) Search for antiviral activity in higher plant extracts. Fitoterapia 59:422-424.

Wagate C, Mbaria J, Gakuya D, Nanyingi M, Kareru P, Njuguna A, Gitahi N, Macharia J, Njonge F (2010) Screening of some Kenyan medicinal plants for antibacterial activity. Phytotherapy Research 24(1):150-153.

Wagoner J, Negash A, Kane O, Martinez I, Nahmias Y, Bourne N, Pécheur E, Graf T, Oberlies N, Lohmann V, CaO F, Tavis J, Polyak S (2010) Multiple effects of silymarin on the hepatitis C virus lifecycle. Hepatology 51(6):1912-1921.

Wegiera M, Smolarz HD, Jedruch M, Korczak M, Koproń K (2012) Cytotoxic effect of some medicinal plants from Asteraceae family on J-45.01 leukemic cell line-pilot study. Acta Poloniae Pharmaceutica 69(2):263-268.

Westfall RE (2003). Galactogogue herbs: A qualitative study and review. Canadian Journal of Midwifery Research and Practice 2(2):22-27.

Wider B, Pittler MH, Thompson-Coon J, Ernst E (2009) Artichoke leaf extract for treating hypercholesterolaemia. Cochrane Database Systematic Reviews 7(4), doi: $10.1002 / 14651858$.

Widrig R, Suter A, Saller R, Melzer J (2007) Choosing between NSAID and arnica for topical treatment of hand osteoarthritis in a randomised, double-blind study. Rheumatology International 27(6):585-591.

Williams C, Harborne J, Geiger H, Hoult J (1999) The flavonoids of Tanacetum parthenium and Tanacetum vulgare and their anti-inflammatory properties. Phytochemistry 51(3):417-423. 
Xie G, Schepetkin I, Quinn M (2007) Immunomodulatory activity of acidic polysaccharides isolated from Tanacetum vulgare. International Immunopharmacology 7(13):1639-1650.

Xie YY, Yuan D, Yang JY, Wang LH, Wu CF (2009) Cytotoxic activity of flavonoids from the flowers of Chrysanthemum morifolium on human colon cancer Colon205 cells. Journal of Asian Natural Products Research 11(9):771-778.

Xu LW, Chen J, Qi HY, Shi YP (2012) Phytochemicals and their biological activities of plants in Tagetes L. Chinese Herbal Medicines 4(2):103-117.

Yaeesh S, Jamal Q, Khan AU, Gilani A (2006) Studies on hepatoprotective, antispasmodic and calcium antagonist activities of the aqueous-methanol extract of Achillea millefolium. Phytotherapy Research 20(7):546-551.

Yarnell E, Abascal K (2009) Dandelion (Taraxacum officinale and Taraxacum mongolicum). Integrative Medicine 8(2):35-38.

Yasukawa K (1991) Sterol and triterpene derivatives from plants inhibit the effect of a tumor promotor, and sitosterol and betulinic acid inhibit tumor formation in mouse skin two-stage carcinogenesis. Oncology 48:72-76.

Yasukawa K, Kitanaka S, Seo S (2002) Inhibitory effect of stevioside on tumor promotion by 12-0-tetradecanoylphorbol-13-acetate in two-stage carcinogenesis in mouse skin. Biological \& Pharmaceutical Bulletin 25:1488-1490.

Yu D, Yuan Y, Jiang L, Tai Y, Yang X, Hu F, Xie Z (2013) Anti-inflammatory effects of essential oil in Echinacea purpurea. Pakistan Journal of Pharmaceutical Sciences 26(2):403-408.

Yukes JE, Balick MJ (2010) Dominican medicinal plants: A guide for health care providers. New York Botanical Garden, New York.

Zaidi S, Muhammad J, Shahryar S, Usmanghani K, Gilani A, Jafri W, Sugiyama T (2012) Anti-inflammatory and cytoprotective effects of selected Pakistani medicinal plants in Helicobacter pylori-infected gastric epithelial cells. Journal of Ethnopharmacology 141(1):403-410.

Zardini EM (1984a) Etnobotánica de Compuestas argentinas, con especial referencia a su uso farmacológico I. Acta Farmacéutica Bonaerense 3(1):77-99.

Zardini EM (1984b) Etnobotánica de Compuestas argentinas, con especial referencia a su uso farmacológico II. Acta Farmacéutica Bonaerense 3(2):169-194.

Zeggwagh NA, Michel JB, Eddouks M (2013) Vascular effects of aqueous extract of Chamaemelum nobile: In vitro pharmacological studies in rats. Clinical and Experimental Hypertension 35(3):200-206.

Zhan T, Digel M, Küch E, Stremmel W, Füllekrug J (2011) Silybin and dehydrosilybin decrease glucose uptake by inhibiting GLUT proteins. Journal of Cellular Biochemistry 112(3):849-859.

Zhu X, Zhang H, Lo R (2004) Phenolic compounds from the leaf extract of artichoke (Cynara scolymus L.) and their antimicrobial activities. Journal of Agricultural and Food Chemistry 52(24):7272-7278. 